TRANSACTIONS OF THE

AMERICAN MATHEMATICAL SOCIETY

Volume 351, Number 6, Pages 2325-2351

S 0002-9947(99)02037-1

Article electronically published on February 16, 1999

\title{
THE ITERATED TRANSFER ANALOGUE OF THE NEW DOOMSDAY CONJECTURE
}

\author{
NORIHIKO MINAMI
}

Dedicated to Professor Dan Kan on the occasion of his retirement from teaching calculus

AbStract. A strong general restriction is given on the stable Hurewicz image of the classifying spaces of elementary abelian $p$-groups. In particular, this implies the iterated transfer analogue of the new doomsday conjecture.

\section{INTRODUCTION}

During the last decade, significant progress has been made in the study of the classifying spaces of elementary abelian $p$-groups, $B V_{s}$, where $V_{s}$ is the rank $s$ elementary abelian $p$-group [C1][Ml][AGM][Ln]. However, these studies dealt with maps from $B V_{s}$, while topologists have been traditionally interested in the stable homotopy of $B V_{s}$ [Ro][KP][Ma2].

Now the purpose of this paper is to give a very general restriction on the $\bmod p$ Hurewicz image of $B V_{s}$. In particular, we will show

Corollary 5.2. The stable Hurewicz map

$$
\pi_{2 n-s}^{s}\left(B V_{s}\right) \rightarrow H_{2 n-s}\left(B V_{s}\right)
$$

has a trivial image, when

$$
\left\{\begin{array}{c}
2^{\nu_{2}(n)+2-2 s}>\nu_{2}(n)+2+\left[\frac{5\left[\frac{s-1}{2}\right]}{2}\right]-\alpha_{2}\left(\left[\frac{s-1}{2}\right]\right) \quad \text { if } p=2, \\
p^{\nu_{p}(n)+2-2 s}>(p-1) \nu_{p}(n)+1+p\left[\frac{s-1}{2}\right]-\alpha_{p}\left(\left[\frac{s-1}{2}\right]\right) \quad \text { if } p \text { is odd. }
\end{array}\right.
$$

Together with the Kahn-Priddy theorem [KP], this result implies the Adams Hopf invariant one theorem [Ad1] as a special case (when $p=2$ and $s=1$ ). Furthermore, when $p=s=2$, this result recovers our previous result [M1], which showed the higher Kervaire invariant one elements $\theta_{j}(j \geq 5)$ are not realized by framed hypersurfaces.

Our result has another implication (which was in fact our motivation). For this purpose, we recall the new doomsday conjecture proposed in [M3]:

Received by the editors November 1, 1996.

1991 Mathematics Subject Classification. Primary 55Q10, 55Q45, 55T15; Secondary 55R12, 55R35, 55N22, 57R77.

Key words and phrases. Adams spectral sequence, transfer, stable homotopy groups of the sphere, Kahn-Priddy theorem, the Kervaire invariant one element.

This work was partially supported by a University of Alabama Research Grant. 
New doomsday Conjecture. For each $s$, there exists some integer $n(s)$ such that no nontrivial element in the image of

$$
\left(\mathcal{P}^{0}\right)^{n(s)}\left(\operatorname{Ext}_{\mathcal{A}_{*}}^{s, *}(\mathbb{Z} / p, \mathbb{Z} / p)\right) \subseteq\left(\operatorname{Ext}_{\mathcal{A}_{*}}^{s, p^{n(s)} *}(\mathbb{Z} / p, \mathbb{Z} / p)\right)
$$

is a nontrivial permanent cycle.

Thus, Corollary 5.2 indicates that at least the iterated transfer analogue of the new doomsday conjecture holds.

To prove our main result (Theorem 5.1), we need the BP-Adams operations [N][Ar2] and the Johnson-Wilson-Yan calculation of $B P_{*}\left(\wedge^{n} P\right)[\mathrm{JW}][\mathrm{JWY}]$, based upon the affirmative solution of the Conner-Floyd conjecture [RW][Mt], just as in our recent papers [M1][M2][M3]. However, the new ingredients here are Wood's theorem [Wo] (formerly Peterson's conjecture $[\mathrm{P}]$ ) and Kameko's reduction $[\mathrm{K}]$ both of which deal with the $\mathcal{A}^{*}$ indecomposable elements of $H^{*}\left(B V_{s}\right)$ (for $p=2$ ).

This paper is organized as follows. In $\S 1$, we recall the results of Wood [Wo] and Kameko $[\mathrm{K}]$, and generalize their results to the odd primary case. In $\S 2$, we use the Adams spectral sequence to show that those relevant elements in $B P_{*}\left(\wedge^{s} P\right)$ have gigantic order. In $\S 3$, we study the action of the $B P$-Adams operations on $B P_{*}\left(\wedge^{s} P\right)$. For this, we need to establish the universal coefficient spectral sequence using the chromatic tower. Our discussion here implies that elements in the $B P$ Hurewicz image $\pi_{*}^{s}\left(\wedge^{s} P\right) \rightarrow B P_{*}\left(\wedge^{s} P\right)$ must have relatively low order. In $\S 4$, we show the transfer homomorphism $H_{n}\left(B V_{s}\right) \rightarrow \operatorname{Ext}_{\mathcal{A}_{*}}^{s, n+s}(\mathbb{Z} / p, \mathbb{Z} / p)$ commutes with the two $S q^{0}$ 's (resp. $\mathcal{P}^{0}$ 's) for $p=2$ (resp. $p$ odd). This material is not used elsewhere in this paper. However, this is philosophically important to relate the iterated transfer to the new doomsday conjecture on the Adams spectral sequence of the sphere. In $\S 5$, we apply our studies in $\S 2$ and $\S 3$ to prove our main result, Theorem 5.1. In $\S 6$, we speculate on a possible way to get around Singer's counterexample [S1] to the naive conjecture concerning the Singer homomorphism.

The author would like to express his gratitude to Peter Landweber, whose careful reading and criticism of a preliminary version of the current paper has been very helpful. His thanks also go to Masaki Kameko, Haynes Miller and Bill Singer for their useful suggestions. Also, the author would like to express his gratitude to mathematicians at the Massachusetts Institute of Topology for their hospitality during the author's visits to MIT during the summer of 1993. Neil Strickland offered the author significant help with emacs. The author's visit to MIT would not have been made possible without the generous support by Dan and Nora Kan, to whom the author expresses his highest gratitude. The travel support to MIT was provided by Alabama EPSCoR.

Notation and convention.

$\mathbb{Z} / p\{g\}$ stands for a group isomorphic to $\mathbb{Z} / p$ with $g$ as its generator.

$H_{*}$ and $H^{*}$ stand for the mod $p$ homology and cohomology, respectively.

$$
P=\Sigma^{\infty} B \mathbb{Z} / p=\Sigma^{\infty} B V_{1} \text {. }
$$

Let $c$ be a fixed generator of $H^{2}\left(\mathbb{C} P^{\infty}\right), y_{2 i} \in H_{2 i}\left(\mathbb{C} P^{\infty}\right)$ be the dual of $c^{i} \in$ $H^{2 i}\left(\mathbb{C} P^{\infty}\right)$, and $x_{2 i+1} \in H_{2 i+1}(P)$ be the image of $y_{2 i}$ under the composite

$$
H_{2 i}\left(\mathbb{C} P^{\infty}\right) \cong H_{2 i+1}\left(\Sigma \mathbb{C} P^{\infty}\right) \rightarrow H_{2 i+1}(P),
$$


where the first map is the suspension isomorphism and the second map is induced by the $S^{1}$-transfer. Let $x_{2 i+2} \in H_{2 i+2}(P)$ be the unique element such that $\beta_{*} x_{2 i+2}=$ $x_{2 i+1}$, where $\beta$ is the Bockstein.

We adopt Milnor's [Mi] description of the dual Steenrod algebra $\mathcal{A}_{*}$ :

$$
\mathcal{A}_{*}=\left\{\begin{array}{clrl}
P\left(\xi_{1}, \xi_{2}, \ldots\right), & \left|\xi_{n}\right|=2^{n}-1, & \text { if } p=2, \\
P\left(\xi_{1}, \xi_{2}, \ldots\right) \otimes E\left(\tau_{0}, \tau_{1}, \ldots\right), & \left|\xi_{n}\right|=2\left(p^{n}-1\right), & \left|\tau_{n}\right|=2 p^{n}-1, \\
& \text { if } p \text { is odd },
\end{array}\right.
$$

where $P$ stands for a polynomial algebra and $E$ stands for an exterior algebra.

$$
\operatorname{Ext}_{\mathcal{A}_{*}^{*}}^{*, *}\left(\mathbb{Z} / p, H_{*}(B P)\right) \cong \operatorname{Ext}_{E_{*}}^{*, *}(\mathbb{Z} / p, \mathbb{Z} / p)=\mathbb{Z} / p\left[u_{0}, u_{1}, \ldots\right],
$$

where $u_{i} \in \operatorname{Ext}_{E_{*}}^{1,2^{i+1}-1}(\mathbb{Z} / p, \mathbb{Z} / p)$ is expressed as $\left[\xi_{i+1}\right]$ in the cobar complex, and corresponds to the usual (Hazewinkel [H] or Araki [Ar1], whichever) generator $v_{i} \in B P_{2^{i+1}-2}$ (resp. $p$ ) when $i \geq 1$ (resp. $\left.i=0\right)$. (Recall $B P_{*}=\mathbb{Z}_{(p)}\left[v_{1}, v_{2}, \ldots\right]$.)

$E\langle k\rangle$ is the exterior quotient Hopf algebra of $\mathcal{A}_{*}$, generated by $\xi_{1}, \ldots, \xi_{k+1}$ (resp. $\left.\tau_{0}, \ldots, \tau_{k}\right)$ for $p=2$ (resp. $p=o d d$ ), whose notation is intended to suggest

$$
\operatorname{Ext}_{\mathcal{A}_{*}^{*}}^{*, *}\left(\mathbb{Z} / p, H_{*}(B P\langle k\rangle)\right) \cong \operatorname{Ext}_{E\langle k\rangle_{*}}^{*, *}(\mathbb{Z} / p, \mathbb{Z} / p)
$$

For simplicity, we write particular spaces of primitive elements as follows:

$$
\begin{gathered}
P B P_{*}(X)=B P \square_{B P_{*} B P} B P_{*}(X), \\
P H_{*}(X)=\mathbb{Z} / p \square_{\mathcal{A}_{*}} H_{*}(X) .
\end{gathered}
$$

\section{WOOD'S THEOREM AND KAMEKO'S REDUCTION}

We use the following numerical functions freely in this section:

$$
\begin{aligned}
& \alpha_{p}(n)=\sum_{i \geq 0} a_{i}, \text { where } n=\sum_{i \geq 0} a_{i} p^{i}, 0 \leq a_{i} \leq p-1 \text { is the } p \text {-adic expansion, } \\
& \beta_{p}(n)=\operatorname{Min}\left\{k \mid n(p-1)=\sum_{1 \leq j \leq k}\left(p^{i_{j}}-1\right) \text { for some } i_{j} \geq 0(1 \leq j \leq k)\right\}, \\
& P_{s}(n)=p n+(p-1) s .
\end{aligned}
$$

Lemma 1.1. (i) $n$ is expressed as $\sum_{1 \leq j \leq m} p^{i_{j}}$ with $i_{j} \geq 0 \Longleftrightarrow \alpha_{p}(n) \leq m \leq n$ and $m \equiv n(\bmod p-1)$.

(ii) $\beta_{p}(n) \leq m \Longleftrightarrow \alpha_{p}(n(p-1)+m) \leq m$.

(iii) $\beta_{p}(n) \leq(p-1) s \Longleftrightarrow \beta_{p}\left(P_{p, s}(n)\right) \leq(p-1) s$.

(iv) $\beta_{p}(n) \leq(p-1) s-1 \Longrightarrow p^{s} \nmid n+s$.

(v) $P_{p, s}^{k}(n)+s=p^{k}(n+s)$.

(vi) $\beta_{p}(n) \leq(p-1) s \Longrightarrow \beta_{p}\left(P_{p, s}^{k}(n)\right)=(p-1) s, \forall k \geq s$.

Proof. (i) Obviously, the largest possible such $m$ is $n$ with $n=n \cdot p^{0}=\underbrace{p^{0}+\cdots+p^{0}}_{n}$.

Now, rearrange $\sum_{1 \leq j \leq m} p^{i_{j}}=\sum_{i \geq 0} b_{i} p^{i}, b_{i} \geq 0$ so that $m=\sum_{i \geq 0} b_{i}$. Any such an expansion is obtained from the trivial expansion $n=\underbrace{p^{0}+\cdots+p^{0}}_{n}$ by repeating the operation $\underbrace{p^{i}+\cdots+p^{i}}_{p} \mapsto p \cdot p^{i}=p^{i+1}$. Of course, each application of this operation reduces the number $m$ by $p-1$, and we can repeat this operation until 
we reach the $p$-adic expansion $n=\sum_{i \geq 0} a_{i} p^{i}, 0 \leq a_{i} \leq p-1$. From this the claim follows.

(ii) This follows from

$$
\begin{aligned}
\beta_{p}(n) \leq m & \Longleftrightarrow n(p-1)=\sum_{1 \leq j \leq m}\left(p^{i_{j}}-1\right) \quad\left(i_{j} \geq 0\right) \\
& \Longleftrightarrow n(p-1)+m=\sum_{1 \leq j \leq m} p^{i_{j}} \quad\left(i_{j} \geq 0\right) \\
& \Longleftrightarrow \alpha_{p}(n(p-1)+m) \leq m,
\end{aligned}
$$

where the last equivalence follows from (i).

(iii) This follows from

$$
\begin{aligned}
(p-1) s \geq \beta_{p}(n) \Longleftrightarrow & (p-1) s \geq \alpha_{p}(n(p-1)+(p-1) s) \\
& =\alpha_{p}(p\{n(p-1)+(p-1) s\}) \\
& =\alpha_{p}(\{p n+(p-1) s\}(p-1)+(p-1) s) \\
\Longleftrightarrow & (p-1) s \geq \beta_{p}(p n+(p-1) s)=\beta_{p}\left(P_{p, s}(n)\right),
\end{aligned}
$$

where we have used (ii) twice.

(iv) From the assumption and (ii), we get $\alpha_{p}(n(p-1)+(p-1) s-1) \leq$ $(p-1) s-1$. Suppose $p^{s} \mid n+s$, contrary to the claim. Then the $p$-adic expansion of $(p-1)(n+s)-1$ begins with

$$
(p-1)(n+s)-1=(p-1)+(p-1) p+\cdots+(p-1) p^{s-1}+\cdots,
$$

which implies $\alpha_{p}((p-1)(n+s)-1) \geq(p-1) s$. Of course, this is a contradiction.

(v) By mathematical induction, we get

$$
\begin{aligned}
P_{p, s}^{k}(n) & =p^{k} n+p^{k-1}(p-1) s+p^{k-2}(p-1) s+\cdots+p(p-1) s+(p-1) s \\
& =p^{k} n+\frac{p^{k}-1}{p-1}(p-1) s=p^{k}(n+s)-s
\end{aligned}
$$

from which the claim follows.

(vi) This follows from (iii), (iv), and (v).

Let $\mathcal{P}$ be the total Steenrod operations:

$$
\mathcal{P}=\left\{\begin{array}{l}
\mathcal{P}^{0}+\mathcal{P}^{1}+\mathcal{P}^{2}+\cdots \quad \text { if } p \text { is odd } \\
S q^{0}+S q^{1}+S q^{2}+\cdots \quad \text { if } p=2
\end{array}\right.
$$

We identify $\mathbb{F}_{p}\left[t_{1}, t_{2}, \ldots, t_{s}\right]$ with the $\mathcal{A}^{*}$ algebra $H^{*}\left(B V_{s}\right)$ and $H^{*}\left(B T^{s}\right)$ for $p=2$ and $p$ odd, respectively. Then, in either case,

$$
\begin{aligned}
\mathcal{P}\left(t_{i}\right) & =t_{i}+t_{i}^{p}, \\
\chi(\mathcal{P})\left(t_{i}\right) & =t_{i}-t_{i}^{p}+t_{i}^{p^{2}}+\cdots+(-1)^{j} t_{i}^{p^{j}}+\cdots
\end{aligned}
$$

for any $1 \leq i \leq s$. Given a monomial $X=t_{1}^{n_{1}} t_{2}^{n_{2}} \cdots t_{s}^{n_{s}}$ in $\mathbb{F}_{p}\left[t_{1}, t_{2}, \ldots, t_{s}\right]$, we denote its degree by $|X|=n_{1}+n_{2}+\cdots+n_{s}$. Notice that this coincides with the usual grading of $H^{*}\left(B V_{s}\right)$ for $p=2$. However, this is one half of the usual grading of $H^{*}\left(B T^{s}\right)$ for $p$ odd. With respect to this grading, both $\mathcal{P}^{i}$ (for $p$ odd) and $S q^{i}$ (for $p=2$ ) increase the degree by $i(p-1)$. The case $p=2$ of the following was first proved by Wood [Wo], who proved a conjecture of Peterson $[\mathrm{P}]$. 
Theorem 1.2. Write down any monomial $X \in \mathbb{F}_{p}\left[t_{1}, t_{2}, \cdots, t_{s}\right]$ of the form

$$
X=t_{1}^{r_{1}} t_{2}^{r_{2}} \cdots t_{s}^{r_{s}} Y^{p}, \quad 0 \leq r_{i} \leq p-1,
$$

for some monomial $Y \in \mathbb{F}_{p}\left[t_{1}, t_{2}, \cdots, t_{s}\right]$. Then, $X$ is $\mathcal{A}^{*}$-decomposable, whenever

$$
\alpha_{p}\left(|X|(p-1)+\left(r_{1}+r_{2}+\cdots r_{s}\right)\right)>r_{1}+r_{2}+\cdots r_{s} .
$$

Proof. We begin by noticing

$$
X=t_{1}^{r_{1}} t_{2}^{r_{2}} \cdots t_{s}^{r_{s}} \cdot \mathcal{P}^{|Y|}(y) \equiv \chi\left(\mathcal{P}^{|Y|}\right)\left(t_{1}^{r_{1}} t_{2}^{r_{2}} \cdots t_{s}^{r_{s}}\right) \cdot Y
$$

modulo $\mathcal{A}^{*}$-decomposables. Suppose, to the contrary, that $X$ is $\mathcal{A}^{*}$-indecomposable. Then, $\chi\left(\mathcal{P}^{|Y|}\right)\left(t_{1}^{r_{1}} t_{2}^{r_{2}} \cdots t_{s}^{r_{s}}\right)$ should be nonzero. However, as

$$
\begin{aligned}
& \chi\left(\mathcal{P}^{|Y|}\right)\left(t_{1}^{r_{1}} t_{2}^{r_{2}} \cdots t_{s}^{r_{s}}\right) \\
= & \text { sum of monomials of the form } \underbrace{t_{1}^{p_{1}^{1}} \cdots t_{1}^{p_{1}^{r_{1}}}}_{r_{1}} \cdots \underbrace{t_{s}^{p_{s}^{i_{s}^{1}} \cdots t_{s}^{p_{s}^{r_{s}}}}}_{r_{s}}
\end{aligned}
$$

and

$$
\left|\chi\left(\mathcal{P}^{|Y|}\right)\left(t_{1}^{r_{1}} t_{2}^{r_{2}} \cdots t_{s}^{r_{s}}\right)\right|=|Y|(p-1)+r_{1}+r_{2}+\cdots r_{s},
$$

$|Y|(p-1)+r_{1}+r_{2}+\cdots r_{s}$ should be expressed as a sum of $\left(r_{1}+r_{2}+\cdots r_{s}\right)$ many $p$-powers (including $\left.1=p^{0}\right)$. From Lemma 1.1(i), this implies

$$
\alpha_{p}\left(|Y|(p-1)+r_{1}+r_{2}+\cdots r_{s}\right) \leq r_{1}+r_{2}+\cdots r_{s} .
$$

But, as

$$
\begin{aligned}
& |X|(p-1)+\left(r_{1}+r_{2}+\cdots r_{s}\right) \\
& \quad=\left\{|Y| p+\left(r_{1}+r_{2}+\cdots r_{s}\right)\right\}(p-1)+\left(r_{1}+r_{2}+\cdots r_{s}\right) \\
& \quad=\left(|Y|(p-1)+\left(r_{1}+r_{2}+\cdots r_{s}\right)\right) p,
\end{aligned}
$$

this is equivalent to

$$
\alpha_{p}\left(|X|(p-1)+\left(r_{1}+r_{2}+\cdots r_{s}\right)\right) \leq\left(r_{1}+r_{2}+\cdots r_{s}\right) .
$$

Of course, this is a contradiction, and the claim is claimed.

Corollary 1.3. The above $X$ is $\mathcal{A}^{*}$-decomposable if $\beta_{p}(|X|)>r_{1}+r_{2}+\cdots r_{s}$.

Proof. This immediately follows from Theorem 1.2 and Lemma 1.1 (ii).

Corollary 1.4. Suppose $Q H^{n}\left(B V_{s}\right) \neq 0$ (resp. $Q H^{2 n}\left(B T^{s}\right) \neq 0$ ) for $p=2$ (resp. $p=$ odd $)$. Then $\beta_{p}(n) \leq s(p-1)\left(\Longleftrightarrow \alpha_{p}((n+s)(p-1)) \leq s(p-1)\right.$ by Lemma 1.1(ii)).

Proof. Suppose, to the contrary, that $\beta_{p}(n)>s(p-1)$. Then, as $s(p-1) \geq r_{1}+$ $r_{2}+\cdots+r_{s}$ in Theorem 1.2, Corollary 1.3 implies any monomial in $H^{n}\left(B V_{s}\right)$ (resp. $H^{2 n}\left(B T^{s}\right)$ ) for $p=2$ (resp. $p=$ odd) is $\mathcal{A}^{*}$-decomposable, i.e. $Q H^{n}\left(B V_{s}\right)=0$ (resp. $\left.Q H^{2 n}\left(B T^{s}\right)=0\right)$ for $p=2$ (resp. $p=$ odd). This is a contradiction.

The following result is used to study $B V_{s}$ when $p$ is odd.

Proposition 1.5. Suppose $p$ is odd. Suppose further that $s=1$ or $\nu_{p}(n) \geq$ $s+\left[\log _{p}\left[\frac{s}{2}\right]\right]+1$ and $s \geq 2$. Then the smash product of the (reduced) $S^{1}$-transfer $\Sigma B T_{+}^{1} \rightarrow P$ induces an isomorphism

$$
P H_{2(n-s)}\left(B T_{+}^{s}\right) \cong P H_{2 n-s}\left(\Sigma^{s} B T_{+}^{s}\right) \rightarrow P H_{2 n-s}\left(\wedge^{s} P\right) .
$$


Proof. Since the case $s=1$ is very easy to see, we assume $s \geq 2$ and $\nu_{p}(n) \geq$ $s+\left[\log _{p}\left[\frac{s}{2}\right]\right]+1$. Then it is enough to show

$$
\left(\mathbb{Z} / p \square_{P_{*}} H_{*}\left(B T_{+}^{s}\right)\right)_{2(n-s)} \cong\left(\mathbb{Z} / p \square_{P_{*}} H_{*}\left(\wedge^{s} P\right)\right)_{2 n-s} .
$$

Since

$$
H_{*}(P) \cong H_{*}\left(\Sigma B T_{+}^{1}\right) \oplus H_{*}\left(\Sigma^{2} B T_{+}^{1}\right),
$$

as a $P_{*}$-comodule, the right-hand side of $(\mathrm{P})$ becomes the direct sum of the left-hand side of $(\mathrm{P})$ and additional $\mathbb{Z} / p$-modules of the form

$$
\left(\mathbb{Z} / p \square_{P_{*}} H_{*}\left(B T_{+}^{s}\right)\right)_{2(n-s-l)}
$$

with $1 \leq l \leq\left[\frac{s}{2}\right]$. We must show these additional modules vanishes. For this, by Corollary 1.4, it is enough to show

$$
\alpha((n-l)(p-1))>s(p-1),
$$

when $\nu_{p}(n) \geq s+\left[\log _{p}\left[\frac{s}{2}\right]\right]+1$ and $1 \leq l \leq\left[\frac{s}{2}\right]$. We write

$$
\begin{gathered}
n=a p^{\nu_{p}(n)}, a \geq 1, \\
l=p^{\left[\log _{p}\left[\frac{s}{2}\right]\right]+1}-b, 0<b<p^{\left[\log _{p}\left[\frac{s}{2}\right]\right]+1} .
\end{gathered}
$$

Then,

$$
\begin{aligned}
(n-l)(p-1) & =a(p-1) p^{\nu_{p}(n)}-(p-1)\left(p^{\left[\log _{p}\left[\frac{s}{2}\right]\right]+1}-b\right) \\
& =\{a(p-1)-1\} p^{\nu_{p}(n)}+\left(p^{\nu_{p}(n)}-p^{\left[\log _{p}\left[\frac{s}{2}\right]\right]+2}\right) \\
& +\left(p^{\left[\log _{p}\left[\frac{s}{2}\right]\right]+1}+b(p-1)\right) .
\end{aligned}
$$

Clearly,

$$
\begin{aligned}
& \alpha_{p}((n-l)(p-1))=\alpha_{p}\left(\{a(p-1)-1\} p^{\nu_{p}(n)}\right) \\
& \quad+\alpha_{p}\left(p^{\nu_{p}(n)}-p^{\left[\log _{p}\left[\frac{s}{2}\right]\right]+2}\right)+\alpha\left(p^{\left[\log _{p}\left[\frac{s}{2}\right]\right]+1}+b(p-1)\right)
\end{aligned}
$$

and

$$
\left\{\begin{aligned}
\alpha_{p}\left(\{a(p-1)-1\} p^{\nu_{p}(n)}\right) & \geq p-2\left(\text { since } \alpha_{p}(a(p-1)-1) \equiv-1(\bmod p-1)\right) \\
\alpha_{p}\left(p^{\nu_{p}(n)}-p^{\left[\log _{p}\left[\frac{s}{2}\right]\right]+2}\right) & =\left(\nu_{p}(n)-\left[\log _{p}\left[\frac{s}{2}\right]\right]-2\right)(p-1) \\
\alpha\left(p^{\left[\log _{p}\left[\frac{s}{2}\right]\right]+1}+b(p-1)\right) & \geq 2 \quad\left(\text { since } 0<b<p^{\left[\log _{p}\left[\frac{s}{2}\right]\right]+1}\right)
\end{aligned}\right.
$$

Therefore,

$$
\alpha_{p}((n-l)(p-1))>\left(\nu_{p}(n)-\left[\log _{p}\left[\frac{s}{2}\right]\right]-1\right)(p-1) \geq s(p-1),
$$

which completes the proof. 
Let $\mathbb{F}_{p}\left[t_{1}, t_{2}, \cdots, t_{s}\right]^{n}$ be the degree $n$-part of $\mathbb{F}_{p}\left[t_{1}, t_{2}, \cdots, t_{s}\right]$. We define a couple of $\mathbb{F}_{p}$-linear maps

$$
\begin{aligned}
\phi: \mathbb{F}_{p}\left[t_{1}, t_{2}, \cdots, t_{s}\right]^{n} & \rightarrow \mathbb{F}_{p}\left[t_{1}, t_{2}, \cdots, t_{s}\right]^{P_{p, s}(n),}, \\
Y & \mapsto t_{1}^{p-1} \cdots t_{s}^{p-1}\left(Y^{p}\right), \\
\psi: \mathbb{F}_{p}\left[t_{1}, t_{2}, \cdots, t_{s}\right]^{P_{p, s}(n)} & \rightarrow \mathbb{F}_{p}\left[t_{1}, t_{2}, \cdots, t_{s}\right]^{n}, \\
X & \mapsto \begin{cases}Y & \text { if } X=\phi(Y), \\
0 & \text { otherwise },\end{cases}
\end{aligned}
$$

where $Y$ and $X$ are monomials.

Lemma 1.6. Suppose $0 \leq r \leq p-1$ and $1 \leq i \leq s$. Then, in the expansion of the total Steenrod operations action

$$
\mathcal{P}\left(t_{i}^{r}\right)=t_{i}^{r}\left(\sum_{o \leq j \leq r}\left(\begin{array}{l}
r \\
j
\end{array}\right) t_{i}^{j(p-1)}\right)=\sum_{o \leq j \leq r}\left(\begin{array}{l}
r \\
j
\end{array}\right) t_{i}^{r+j(p-1)},
$$

the degree $r+j(p-1)$ of a typical term is congruent to $p-1$ modulo $p$, only if $r=p-1$ and $j=0$.

Proof. This immediately follows from $0 \leq j \leq r \leq p-1$ and $r-j \equiv r+j(p-1) \equiv$ $p-1(\bmod p)$.

Proposition 1.7. (i) $\psi \mathcal{P} X=\mathcal{P} \psi X$ for any monomial $X$. In fact,

$$
\psi\left(\mathcal{P}^{a} X\right)=\left\{\begin{array}{cl}
\mathcal{P}^{\frac{a}{p}}(\psi X) & \text { if } p \mid a, \\
0 & \text { otherwise. }
\end{array}\right.
$$

(ii) The kernel of the composition

$$
\mathbb{F}_{p}\left[t_{1}, t_{2}, \cdots, t_{s}\right]^{P_{p, s}(n) \stackrel{\psi}{\rightarrow}} \mathbb{F}_{p}\left[t_{1}, t_{2}, \cdots, t_{s}\right]^{n} \stackrel{Q}{\rightarrow} Q \mathbb{F}_{p}\left[t_{1}, t_{2}, \cdots, t_{s}\right]^{n}
$$

is the sub $\mathbb{F}_{p}$-vector space generated by $\operatorname{Ker} \psi$ and the $\mathcal{A}^{*}$-decomposable elements.

Proof. (i) Write down any monomial $X \in \mathbb{F}_{p}\left[t_{1}, t_{2}, \cdots, t_{s}\right]$ in the form

$$
X=t_{1}^{r_{1}} t_{2}^{r_{2}} \cdots t_{s}^{r_{s}} Y^{p}, \quad 0 \leq r_{i} \leq p-1,
$$

for some monomial $Y \in \mathbb{F}_{p}\left[t_{1}, t_{2}, \cdots, t_{s}\right]$. Notice that $X=\phi\left(Y^{\prime}\right)$ for some monomial $Y^{\prime}$ if and only if $r_{i}=p-1$ for any $1 \leq i \leq s$. Then

$$
\begin{aligned}
\psi \mathcal{P} X & =\psi\left(\mathcal{P}\left(t_{1}^{r_{1}} t_{2}^{r_{2}} \cdots t_{s}^{r_{s}} Y^{p}\right)\right) \\
& =\psi\left(\mathcal{P}\left(t_{1}\right)^{r_{1}} \mathcal{P}\left(t_{2}\right)^{r_{2}} \cdots \mathcal{P}\left(t_{s}\right)^{r_{s}}(\mathcal{P} Y)^{p}\right) \\
& = \begin{cases}\mathcal{P} Y & \text { if } r_{i}=p-1 \text { for any } 1 \leq i \leq s, \\
0 & \text { otherwise }\end{cases} \\
& =\mathcal{P} \psi\left(t_{1}^{r_{1}} t_{2}^{r_{2}} \cdots t_{s}^{r_{s}} Y^{p}\right) \\
& =\mathcal{P} \psi X,
\end{aligned}
$$

where we used Lemma 1.6 in the second equality. The second claim follows from this and a degree observation based on $P_{p, s}\left(\frac{a}{p}+|\psi X|\right)=a+P_{p, s}(|\psi X|)$. 
(ii) Suppose $A \in \mathbb{F}_{p}\left[t_{1}, t_{2}, \cdots, t_{s}\right]^{P_{p, s}(n)}$ is contained in the kernel of the composite $Q \circ \psi$. Then, we may write

$$
\psi(A)=\sum_{i} \lambda_{i} \mathcal{P}^{b_{i}} Y_{i}
$$

for some $\lambda_{i} \in \mathbb{F}_{p}, b_{i}>0$, and monomials $Y_{i}$. But, by (i), the right-hand side becomes $\psi\left(\sum_{i} \lambda_{i} \mathcal{P}^{b_{i}}\left(\phi\left(Y_{i}\right)\right)\right)$. Therefore,

$$
A-\sum_{i} \lambda_{i} \mathcal{P}^{b_{i}}\left(\phi\left(Y_{i}\right)\right) \in \operatorname{Ker} \psi \quad\left(b_{i}>0\right),
$$

from which the claim follows.

Now we are ready to state and prove Kameko's reduction $[\mathrm{K}]$ for any prime $p$. ([K] considered only the case $p=2$.)

Theorem 1.8. (i) $\psi$ induces a surjective $\mathbb{F}_{p}$-linear map

$$
Q \psi: Q \mathbb{F}_{p}\left[t_{1}, t_{2}, \cdots, t_{s}\right]^{P_{p, s}(n)} \rightarrow Q \mathbb{F}_{p}\left[t_{1}, t_{2}, \cdots, t_{s}\right]^{n} .
$$

(ii) If $\beta_{p}\left(P_{p, s}(n)\right)=s(p-1)$, this is an isomorphism.

Proof. (i) $\psi$ induces $Q \psi$ by Proposition 1.7 (i). $Q \psi$ is clearly surjective as $\psi$ is surjective (with $\phi$ as its section).

(ii) From Proposition 1.7 (ii), the kernel of the composition

$$
\mathbb{F}_{p}\left[t_{1}, t_{2}, \cdots, t_{s}\right]^{P_{p, s}(n)} \stackrel{\psi}{\rightarrow} \mathbb{F}_{p}\left[t_{1}, t_{2}, \cdots, t_{s}\right]^{n} \stackrel{Q}{\rightarrow} Q \mathbb{F}_{p}\left[t_{1}, t_{2}, \cdots, t_{s}\right]^{n}
$$

is the sub $\mathbb{F}_{p}$-vector space generated by $\operatorname{Ker} \psi$ and the $\mathcal{A}^{*}$-decomposable elements. Now Ker $\psi$ is generated by those monomials

$$
X=t_{1}^{r_{1}} t_{2}^{r_{2}} \cdots t_{s}^{r_{s}} Y^{p}, \quad 0 \leq r_{i} \leq p-1
$$

with some monomial $Y \in \mathbb{F}_{p}\left[t_{1}, t_{2}, \cdots, t_{s}\right]$, such that $\sum_{1 \leq i \leq s} r_{i}<s(p-1)=$ $\beta_{p}(|X|)$. Then, $X$ is $\mathcal{A}^{*}$-decomposable by Corollary 1.3. This implies that $\operatorname{Ker} Q \circ \psi$ consists of the $\mathcal{A}^{*}$-decomposable elements, from which the result follows.

Corollary 1.9. (i) For $p=2, \psi$ induces a surjective $\mathbb{F}_{2}$-linear map

$$
Q \psi: Q H^{P_{p, s}(n)}\left(B V_{s}\right) \rightarrow Q H^{n}\left(B V_{s}\right) .
$$

If $\beta_{p}\left(P_{p, s}(n)\right)=\beta_{p}(2 n+s)=s$, this is an isomorphism.

(ii) For $p=$ odd, $\psi$ induces a surjective $\mathbb{F}_{p}$-linear map

$$
Q \psi: Q H^{2 P_{p, s}(n)}\left(B T^{s}\right) \rightarrow Q H^{2 n}\left(B T^{s}\right) .
$$

If $\beta_{p}\left(P_{p, s}(n)\right)=\beta_{p}(p n+s(p-1))=s(p-1)$, this is an isomorphism.

Corollary 1.9(i) is the original Kameko's reduction $[\mathrm{K}]$. Now the following is the dual statement.

Corollary 1.10. (i) When $p=2$, we have a well-defined injective $\mathbb{F}_{2}$-linear map

$$
\begin{aligned}
S q^{0}: P H_{n}\left(B V_{s}\right) & \rightarrow P H_{P_{p, s}(n)}\left(B V_{s}\right), \\
x_{i_{1}} \otimes x_{i_{2}} \otimes \cdots \otimes x_{i_{s}} & \mapsto x_{2 i_{1}+1} \otimes x_{2 i_{2}+1} \otimes \cdots \otimes x_{2 i_{s}+1} .
\end{aligned}
$$

If $\beta_{p}\left(P_{p, s}(n)\right)=s$, this is an isomorphism. 
(ii) When $p=$ odd, we have a well-defined injective $\mathbb{F}_{p}$-linear map

$$
\begin{aligned}
\mathcal{P}^{0}: P H_{2 n}\left(B T^{s}\right) & \rightarrow P H_{2 P_{p, s}(n)}\left(B T^{s}\right), \\
y_{2 i_{1}} \otimes y_{2 i_{2}} \otimes \cdots \otimes y_{2 i_{s}} & \mapsto y_{2\left(p i_{1}+p-1\right)} \otimes y_{2\left(p i_{2}+p-1\right)} \otimes \cdots \otimes y_{2\left(p i_{s}+p-1\right)} .
\end{aligned}
$$

If $\beta_{p}\left(P_{p, s}(n)\right)=s(p-1)$, this is an isomorphism.

Corollary 1.11. For any $l \geq 0$,

$$
\left\{\begin{aligned}
\left(S q^{0}\right)^{l}: P H_{2^{s-1} t-s}\left(B V_{s}\right) & \rightarrow P H_{2^{s+l-1} t-s}\left(B V_{s}\right), \\
\left(\mathcal{P}^{0}\right)^{l}: P H_{2\left(p^{s-1} t-s\right)}\left(B T^{s}\right) & \rightarrow P H_{2\left(p^{s+l-1} t-s\right)}\left(B T^{s}\right)
\end{aligned}\right.
$$

is an isomorphism.

Proof. In both cases, we may assume $\beta_{p}(t-s) \leq(p-1) s$, because, otherwise, both the source and the target are trivial by Corollary 1.4 and Lemma 1.1 (iii). But, if $\beta_{p}(t-s) \leq(p-1) s$, the claim is an immediate consequence of Corollary 1.10 and Lemma 1.1 (vi).

To conclude this section, we make the following generalization for an odd prime $p$ :

$$
\begin{aligned}
& \mathcal{P}^{0}: P H_{n}\left(B V_{s}\right) \rightarrow P H_{P_{p, s}(n)}\left(B V_{s}\right),
\end{aligned}
$$

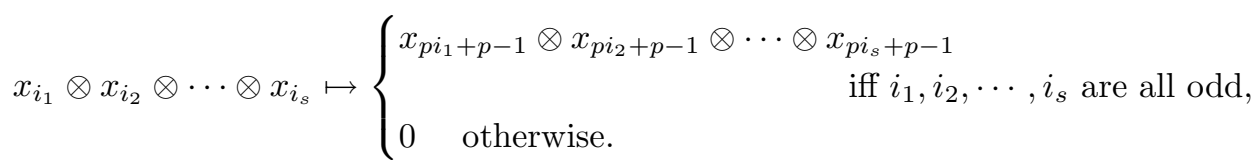

The well-definedness of this $\mathcal{P}^{0}$ may be easily verified, using Corollary 1.10 (ii) and the $P_{*}$-comodule isomorphism

$$
H_{*}(P) \cong H_{*}\left(\Sigma B T_{+}^{1}\right) \oplus H_{*}\left(\Sigma^{2} B T_{+}^{1}\right) .
$$

\section{The Adams spectral Sequence of $B P_{*}\left(\wedge^{s} P\right)$}

To study $B P_{*}\left(B V_{s}\right)$, recall that the canonical projection

$$
\begin{aligned}
B V_{s}=(B \mathbb{Z} / p) \times(B \mathbb{Z} / p) \times \cdots \times( & (B \mathbb{Z} / p) \\
& \rightarrow(B \mathbb{Z} / p) \wedge(B \mathbb{Z} / p) \wedge \cdots \wedge(B \mathbb{Z} / p)=\wedge^{s} P
\end{aligned}
$$

stably splits so that $\wedge^{s} P$ constitutes the essential part of $B V_{s}: x_{i_{1}} \otimes x_{i_{2}} \otimes \cdots \otimes$ $x_{i_{s}} \in H_{*}\left(B V_{s+}\right)$, where $i_{1}, i_{2}, \cdots, i_{s} \geq 0$, is contained in $H_{*}\left(\wedge^{s} P\right)$ if and only if $i_{1}, i_{2}, \cdots, i_{s} \geq 1$.

Therefore, we are going to study $B P_{*}\left(\wedge^{s} P\right)$, which still has a very complicated additive structure. Fortunately, as was noticed by $[\mathrm{JWY}][\mathrm{JW}]$, the affirmative solution $[\mathrm{RW}][\mathrm{Mt}]$ of the Conner-Floyd conjecture implies that its classical Adams spectral sequence collapses at its $E_{2}$-term, and this allows us to use more tractable $\operatorname{Ext}_{E\langle s\rangle_{*}^{*}}^{*, *}\left(\mathbb{Z} / p, H_{*}\left(\wedge^{s} P\right)\right)$, instead of $B P_{*}\left(\wedge^{s} P\right)$, to evaluate the $B P_{*}-$ order of those elements we are interested in. (Note that the multiplication by $p$ in $B P_{*}\left(\wedge^{s} P\right)$ corresponds to the multiplication by $u_{0} \in \operatorname{Ext}_{E\langle s\rangle_{*}}^{1,1}(\mathbb{Z} / p, \mathbb{Z} / p)$ in $\operatorname{Ext}_{E\langle s\rangle_{*}}^{*, *}\left(\mathbb{Z} / p, H_{*}\left(\wedge^{s} P\right)\right)$.) We begin with a summary of known results, which are necessary in our approach: 
Proposition 2.1. (i) The Adams spectral sequence

$$
\operatorname{Ext}_{\mathcal{A}_{*}^{* *}}^{*}\left(\mathbb{Z} / p, H_{*}\left(B P \wedge \wedge^{s} P\right)\right) \Longrightarrow B P_{*}\left(\wedge^{s} P\right)
$$

collapses.

(ii) $A s \mathbb{Z} / p\left[u_{s}\right]$-modules,

$$
\operatorname{Ext}_{E\langle s\rangle_{*}}^{*, *}\left(\mathbb{Z} / p, H_{*}\left(\wedge^{s} P\right)\right)=\operatorname{Ext}_{E\langle s-1\rangle_{*}}^{*, *}\left(\mathbb{Z} / p, H_{*}\left(\wedge^{s} P\right)\right) \otimes \mathbb{Z} / p\left[u_{s}\right] .
$$

(iii) $\operatorname{Ext}_{E\langle s\rangle_{*}}^{* * *}\left(\mathbb{Z} / p, H_{*}\left(\wedge^{s} P\right)\right)$ contains a sub $\mathbb{Z} / p\left[u_{0}, u_{1}, \cdots, u_{s}\right]$-module

$$
\bigoplus_{k_{1}, \cdots, k_{s} \geq 1} \mathbb{Z} / p\left\{x_{2 k_{1}-1} \otimes x_{2 k_{2}-1} \otimes \cdots \otimes x_{2 k_{s}-1}\right\} \otimes \mathbb{Z} / p\left[u_{s}\right],
$$

where $x_{2 k_{1}-1} \otimes x_{2 k_{2}-1} \otimes \cdots \otimes x_{2 k_{s}-1} \in \operatorname{Ext}_{E s_{*}}^{0,2 \sum_{1 \leq i \leq s} k_{i}-s}\left(\mathbb{Z} / p, H_{*}\left(\wedge^{s} P\right)\right)$.

(iv) There is a canonical identification of the above $\mathbb{Z} / p\left[u_{0}, u_{1}, \ldots, u_{s}\right]$ submodules of $\operatorname{Ext}_{E\langle s\rangle_{*}^{*}}^{*, *}\left(\mathbb{Z} / p, H_{*}\left(\wedge^{s} P\right)\right)$ as $\otimes_{\mathbb{Z} / p\left[u_{0}, u_{1}, \ldots, u_{s}\right]}^{s} \operatorname{Ext}_{E\langle s\rangle_{*}}^{*, *}\left(\mathbb{Z} / p, H_{*}(P)\right)$ :

$$
\begin{aligned}
i: \bigoplus_{k_{i} \geq 1} \mathbb{Z} / p\left\{x_{2 k_{1}-1} \otimes x_{2 k_{2}-1} \otimes \cdots \otimes\right. & \left.x_{2 k_{s}-1}\right\} \otimes \mathbb{Z} / p\left[u_{s}\right] \\
& \rightarrow \bigotimes_{\mathbb{Z} / p\left[u_{0}, u_{1}, \ldots, u_{s}\right]}^{s} \operatorname{Ext}_{E\langle s\rangle_{*}}^{*, *}\left(\mathbb{Z} / p, H_{*}(P)\right), \\
x_{2 k_{1}-1} \otimes x_{2 k_{2}-1} \otimes \cdots \otimes x_{2 k_{s}-1} & \mapsto x_{2 k_{1}-1} \otimes x_{2 k_{2}-1} \otimes \cdots \otimes x_{2 k_{s}-1} .
\end{aligned}
$$

Proof. For (i)(ii), see [JWY][JW]. The first part of (iii) follows from (i)(ii) and the solution of the Conner-Floyd conjecture [RW][Mt], which was also used in (i) [JWY]. The second part of (iii) about the splitting is proved in [JWY].

For (iv), we first notice that $\bigotimes_{\mathbb{Z} / p\left[u_{0}, u_{1}, \ldots, u_{s}\right]}^{s} \operatorname{Ext}_{E\langle s\rangle_{*}}^{*, *}\left(\mathbb{Z} / p, H_{*}(P)\right)$ is generated by $\bigoplus_{k_{1}, \cdots, k_{s}} \mathbb{Z} / p\left\{x_{2 k_{1}-1} \otimes x_{2 k_{2}-1} \otimes \cdots \otimes x_{2 k_{s}-1}\right\}$, as a $\mathbb{Z} / p\left[u_{0}, u_{1}, \ldots, u_{s}\right]$-module. This is because such is the case for each tensor factor $\operatorname{Ext}_{E\langle s\rangle_{*}}^{* * *}\left(\mathbb{Z} / p, H_{*}(P)\right)[\mathrm{D}]$. So, thanks to (iii), the claim would follow if we could canonically embed

$$
\bigotimes_{\mathbb{Z} / p\left[u_{0}, u_{1}, \ldots, u_{s}\right]}^{s} \operatorname{Ext}_{E\langle s\rangle_{*}}^{*, *}\left(\mathbb{Z} / p, H_{*}(P)\right) \quad \text { in } \operatorname{Ext}_{E\langle s\rangle_{*}}^{*, *}\left(\mathbb{Z} / p, H_{*}\left(\wedge^{s} P\right)\right) .
$$

But this follows immediately from the Ext-analogue (see the proof of Proposition 2.1 (iv) in [M3]) of Landweber's bordism Künneth theorem [L1], which latter enables us to embed $\bigotimes_{B P_{*}}^{s} B P_{*}(P)$ in $B P_{*}\left(\wedge^{s} P\right)[\mathrm{JW}]$.

To understand the order of those relevant elements in $B P_{*}\left(\wedge^{s} P\right)$, we need to know the formula of $u_{0}$-action on

$$
\bigoplus_{k_{1}, \cdots, k_{s}} \mathbb{Z} / p\left\{x_{2 k_{1}-1} \otimes x_{2 k_{2}-1} \otimes \cdots \otimes x_{2 k_{s}-1}\right\} \otimes \mathbb{Z} / p\left[u_{s}\right] .
$$

For a technical reason, we can minimize our task by writing down all the actions of $u_{1}, \ldots, u_{s-1}$ simultaneously. For this purpose, we denote the $\mathbb{Z} / p\left[u_{s}\right]$-module map $x_{2 k_{1}-1} \otimes x_{2 k_{2}-1} \otimes \cdots \otimes x_{2 k_{s}-1} \longmapsto u_{j} x_{2\left(k_{1}-r_{1}\right)-1} \otimes x_{2\left(k_{2}-r_{2}\right)-1} \otimes \cdots \otimes x_{2\left(k_{s}-r_{s}\right)-1}$ by $u_{j} X_{1}^{r_{1}} \cdots X_{s}^{r_{s}}$, and define a power series

$$
f(X)=\left(1+X^{p-1}+X^{p^{2}-1}+\cdots+X^{p^{k}-1} \cdots\right)^{-1} .
$$


Furthermore, using the $p$-adic expansion of $n$,

$$
n=e_{m} p^{m}+e_{m-1} p^{m-1}+\cdots+e_{1} p+e_{0} \quad\left(0 \leq e_{j} \leq p-1 \text { for } 0 \leq j \leq m\right),
$$

we set

$$
\begin{aligned}
\alpha_{p}(n) & =e_{0}+e_{1}+\cdots+e_{m}, \\
\Lambda & =\{0\} \cup\left\{n \mid \alpha_{p}(n)=p-1\right\}, \\
C_{p}(n) & = \begin{cases}-\frac{\alpha_{p}(n) !}{e_{1} ! e_{2} ! \cdots e_{m} !} & \text { when } n>0, \\
1 & \text { when } n=0 .\end{cases}
\end{aligned}
$$

Notice that $C_{p}(n)=\left(e_{1} ! e_{2} ! \cdots e_{m} !\right)^{-1}$ if $n \in \Lambda$ (we use the usual convention $\left.0 !=1\right)$.

Lemma 2.2. $f(X)=\sum_{n \in \Lambda} C_{p}(n) X^{n}$.

Proof. This follows from

$$
\begin{aligned}
f(X) & =\left(1+X^{p-1}+X^{p^{2}-1}+\cdots+X^{p^{k}-1}+\cdots\right)^{-1} \\
& =1-X^{p-1}\left(1+X^{p-1}+X^{p^{2}-1}+\cdots+X^{p^{k}-1}+\cdots\right)^{p-1} \\
& =1-\left(X+X^{p}+X^{p^{2}}+\cdots+X^{p^{k}}+\cdots\right)^{p-1} \\
& =1+\sum_{\alpha_{p}(n)=p-1} C_{p}(n) X^{n}=\sum_{n \in \Lambda} C_{p}(n) X^{n} .
\end{aligned}
$$

Now, the following is the action formula:

Proposition 2.3. The $\mathbb{Z} / p\left[u_{0}, u_{1}, \ldots, u_{s}\right]$-module structure of

$$
\bigoplus_{k_{1}, \cdots, k_{s} \geq 1} \mathbb{Z} / p\left\{x_{2 k_{1}-1} \otimes x_{2 k_{2}-1} \otimes \cdots \otimes x_{2 k_{s}-1}\right\} \otimes \mathbb{Z} / p\left[u_{s}\right]
$$

is determined by the following equalities of $\mathbb{Z} / p\left[u_{s}\right]$-module self-maps:

$$
\begin{aligned}
u_{i} & =u_{s} \cdot \text { total degree } p^{s}-p^{i} \text {-part of } \prod_{1 \leq j \leq s} f\left(X_{j}\right) \\
& =u_{s} \cdot \sum_{r_{1}, \cdots, r_{s} \in \Lambda, r_{1}+\cdots, r_{s}=p^{s}-p^{i}} C_{p}\left(r_{1}\right) \cdots C_{p}\left(r_{s}\right) X_{1}^{r_{1}} \cdots X_{s}^{r_{s}},
\end{aligned}
$$

for any $0 \leq i \leq s$.

Proof. As these actions of $u_{0}, u_{1}, \ldots, u_{s}$ clearly commute, they together define a $\mathbb{Z} / p\left[u_{0}, u_{1}, \ldots, u_{s}\right]$-module structure. Now, we claim these (proposed) actions satisfy

$$
u_{0}+u_{1} X_{j}^{p-1}+u_{2} X_{j}^{p^{2}-1}+\cdots+u_{s} X_{j}^{p^{s}-1}=0 \quad(1 \leq \forall j \leq s) .
$$


By symmetry, it is enough to show this for $j=1$. Since

$$
\begin{aligned}
& u_{0}+u_{1} X_{1}^{p-1}+u_{2} X_{1}^{p^{2}-1}+\cdots+u_{s} X_{1}^{p^{s}-1} \\
= & u_{s}\left(\left(1+X_{1}^{p-1}+X_{1}^{p^{2}-1}+\cdots+X_{1}^{p^{s}-1}+\cdots\right) f\left(X_{1}\right) \prod_{2 \leq j \leq s} f\left(X_{j}\right)\right. \\
& \left.-\left(X_{1}^{p^{s+1}-1}+\cdots\right) \prod_{1 \leq j \leq s} f\left(X_{j}\right)\right)_{\text {total degree } p^{s}-1} \\
= & u_{s}\left(\prod_{2 \leq j \leq s} f\left(X_{j}\right)\right)_{\text {total degree } p^{s}-1}
\end{aligned}
$$

it is enough to show $\left(\prod_{2 \leq j \leq n} f\left(X_{j}\right)\right)_{\text {total degree } p^{s}-1}=0$. However, as the total degree, say $n$, of any nonzero term in the expansion of $\prod_{2 \leq j \leq s} f\left(X_{j}\right)$ satisfies $\alpha_{p}(n) \leq(s-1)(p-1)$ by Lemma 2.2 , this simply follows from $\alpha_{p}\left(p^{s}-1\right)=s(p-1)$. Thus, we have verified (C).

Notice that $(\mathrm{C})$ is just the characterizing property of the $\mathbb{Z} / p\left[u_{0}, u_{1}, \ldots, u_{s}\right]$ module structure on $\mathbb{\bigotimes}_{\mathbb{Z} / p\left[u_{0}, u_{1}, \ldots, u_{s}\right]}^{s} \operatorname{Ext}_{E\langle s\rangle_{*}}^{*, *}\left(\mathbb{Z} / p, H_{*}(P)\right)$. The point is that $\operatorname{Ext}_{E\langle s\rangle_{*}}^{*, *}\left(\mathbb{Z} / p, H_{*}(P)\right)$ is generated by $\left\{x_{2 i-1}\right\}_{i \in \mathbb{N}}$ with the relations

$$
\left\{u_{0} x_{2 k-1}+u_{1} x_{2(k-(p-1))-1}+u_{2} x_{2\left(k-\left(p^{2}-1\right)\right)-1}+\cdots+u_{s} x_{2\left(k-\left(p^{s}-1\right)\right)-1}\right\}_{k \in \mathbb{N}}
$$

(i.e. $u_{0}+u_{1} X^{p-1}+u_{2} X^{p^{2}-1}+\cdots+u_{s} X^{p^{s}-1}=0$ ) as a $\mathbb{Z} / p\left[u_{0}, u_{1}, \ldots, u_{s}\right]$-module [D]. Hence,

$$
\bigotimes_{\mathbb{Z} / p\left[u_{0}, u_{1}, \ldots, u_{s}\right]}^{s} \operatorname{Ext}_{E\langle s\rangle_{*}}^{*, *}\left(\mathbb{Z} / p, H_{*}(P)\right)
$$

is generated as a $\mathbb{Z} / p\left[u_{0}, u_{1}, \ldots, u_{s}\right]$-module by $\left\{x_{2 k_{1}-1} \otimes x_{2 k_{2}-1} \otimes \cdots \otimes x_{2 k_{s}-1}\right\}_{k_{i} \geq 1}$ subject to $(\mathrm{C})$. Therefore, we can define a $\mathbb{Z} / p\left[u_{0}, u_{1}, \ldots, u_{s}\right]$-module homomorphism

$$
\begin{aligned}
h: \bigotimes_{\mathbb{Z} / p\left[u_{0}, u_{1}, \ldots, u_{s}\right]}^{s} \operatorname{Ext}_{E\langle s\rangle_{*}}^{*, *}\left(\mathbb{Z} / p, H_{*}(P)\right) & \\
& \rightarrow\left(\bigoplus_{k_{i} \geq 1} \mathbb{Z} / p\left\{x_{2 k_{1}-1} \otimes x_{2 k_{2}-1} \otimes \cdots \otimes x_{2 k_{s}-1}\right\} \otimes \mathbb{Z} / p\left[u_{s}\right]\right)^{\prime}
\end{aligned}
$$

so that

$$
h\left(x_{2 k_{1}-1} \otimes x_{2 k_{2}-1} \otimes \cdots \otimes x_{2 k_{s}-1}\right)=x_{2 k_{1}-1} \otimes x_{2 k_{2}-1} \otimes \cdots \otimes x_{2 k_{s}-1}
$$

for any $k_{i} \geq 1$, where $\left(\bigoplus_{k_{i} \geq 1} \mathbb{Z} / p\left\{x_{2 k_{1}-1} \otimes x_{2 k_{2}-1} \otimes \cdots \otimes x_{2 k_{s}-1}\right\} \otimes \mathbb{Z} / p\left[u_{s}\right]\right)^{\prime}$ is $\bigoplus_{k_{i}>1} \mathbb{Z} / p\left\{x_{2 k_{1}-1} \otimes x_{2 k_{2}-1} \otimes \cdots \otimes x_{2 k_{s}-1}\right\} \otimes \mathbb{Z} / p\left[u_{s}\right]$ equipped with the proposed $\mathbb{Z} / p\left[u_{0}, u_{1}, \ldots, u_{s}\right]$-module structure. 
On the other hand, in Proposition 2.1 (iv), we constructed a $\mathbb{Z} / p\left[u_{0}, u_{1}, \ldots, u_{s}\right]$ module homomorphism

$$
\begin{aligned}
i: \bigoplus_{k_{i} \geq 1} \mathbb{Z} / p & \left\{x_{2 k_{1}-1} \otimes x_{2 k_{2}-1} \otimes \cdots \otimes x_{2 k_{s}-1}\right\} \otimes \mathbb{Z} / p\left[u_{s}\right] \\
& \rightarrow \bigotimes_{\mathbb{Z} / p\left[u_{0}, u_{1}, \ldots, u_{s}\right]}^{s} \operatorname{Ext}_{E\langle s\rangle_{*}}^{* * *}\left(\mathbb{Z} / p, H_{*}(P)\right)
\end{aligned}
$$

such that $i\left(x_{2 k_{1}-1} \otimes x_{2 k_{2}-1} \otimes \cdots \otimes x_{2 k_{s}-1}\right)=x_{2 k_{1}-1} \otimes x_{2 k_{2}-1} \otimes \cdots \otimes x_{2 k_{s}-1}$ for any $k_{i} \geq 1$. Then, clearly,

$$
\begin{aligned}
h \circ i: & \bigoplus_{k_{i} \geq 1} \mathbb{Z} / p\left\{x_{2 k_{1}-1} \otimes x_{2 k_{2}-1} \otimes \cdots \otimes x_{2 k_{s}-1}\right\} \otimes \mathbb{Z} / p\left[u_{s}\right] \\
& \longrightarrow\left(\bigoplus_{k_{i} \geq 1} \mathbb{Z} / p\left\{x_{2 k_{1}-1} \otimes x_{2 k_{2}-1} \otimes \cdots \otimes x_{2 k_{s}-1}\right\} \otimes \mathbb{Z} / p\left[u_{s}\right]\right)^{\prime}
\end{aligned}
$$

is a $\mathbb{Z} / p\left[u_{0}, u_{1}, \ldots, u_{s}\right]$-module isomorphism which induces the identity map on the underlying set. This immediately implies that the proposed $\mathbb{Z} / p\left[u_{0}, u_{1}, \ldots, u_{s}\right]$ module structure on $\bigoplus_{k_{i} \geq 1} \mathbb{Z} / 2\left\{x_{2 k_{1}-1} \otimes x_{2 k_{2}-1} \otimes \cdots \otimes x_{2 k_{s}-1}\right\} \otimes \mathbb{Z} / p\left[u_{s}\right]$ is the right one.

Now, define the usual lexicographical order among monomials in $H_{*}\left(B V_{s}\right)$ :

$$
\begin{aligned}
& x_{a_{1}} \otimes x_{a_{2}} \otimes \cdots \otimes x_{a_{s}} \prec x_{b_{1}} \otimes x_{b_{2}} \otimes \cdots \otimes x_{b_{s}} \\
\Longleftrightarrow & a_{u}=b_{u}, u<t \text { and } a_{t}<b_{t}, \text { for some } t(1 \leq t \leq s) .
\end{aligned}
$$

Then, the $i=0$ case of Proposition 2.3 immediately implies

Corollary 2.4. ([JWY] 2.11)

$$
\begin{aligned}
& u_{0}^{l}\left(x_{2 k_{1}-1} \otimes x_{2 k_{2}-1} \otimes \cdots \otimes x_{2 k_{s}-1}\right) \\
& =(-1)^{s l} u_{s}^{l}\left(x_{2\left(k_{1}-l\left(p^{s}-p^{s-1}\right)\right)-1} \otimes x_{2\left(k_{2}-l\left(p^{s-1}-p^{s-2}\right)\right)-1} \otimes \cdots \otimes x_{2\left(k_{s}-l(p-1)\right)-1}\right. \\
& \quad+\text { sum of monomials with higher order }) .
\end{aligned}
$$

Proof. It is enough to show this for $l=1$. Then the claim is an immediate consequence of Proposition 2.3, because $\alpha_{p}\left(p^{s}-1\right)=s(p-1)$ and

$$
C_{p}\left(p^{s}-p^{s-1}\right) C_{p}\left(p^{s-1}-p^{s-2}\right) \cdots C_{p}(p-1)=C_{p}(p-1)^{s}=(-1)^{s} .
$$

Proposition 2.5. (i) Suppose $p=2$. Then, if the Thom reduction

$$
B P_{2^{l+s-1} t-s}\left(B V_{s}\right) \rightarrow H_{2^{l+s-1} t-s}\left(B V_{s}\right)
$$

image of an element $\Theta$ is nontrivial and contained in $P H_{2^{l+s-1} t-s}\left(B V_{s}\right)$, the 2exponent of $\Theta$ is at least $2^{l-s}$.

(ii) Suppose $p=$ odd, and $l \geq\left[\log _{p}\left[\frac{s}{2}\right]\right]$ when $s \geq 2$. Then, if the Thom reduction

$$
B P_{2 p^{l+s-1} t-s}\left(B V_{s}\right) \rightarrow H_{2 p^{l+s-1} t-s}\left(B V_{s}\right)
$$

image of $\Theta$ is nontrivial and contained in $\mathrm{PH}_{2 p^{l+s-1} t-s}\left(B V_{s}\right)$, the p-exponent of $\Theta$ is at least $\frac{p^{l-s+1}-1}{p-1}+1$. 
Proof. (i) From Corollary 1.11, $P H_{2^{s+l-1} t-s}\left(B V_{s}\right)=\left(S q^{0}\right)^{l}\left(P H_{2^{s-1} t-s}\left(B V_{s}\right)\right)$. Then, we may assume $\Theta \in B P_{2^{l+s-1} t-s}\left(\wedge^{s} P\right)$, because $S q^{0}\left(H_{*}\left(B V_{s}\right)\right) \subseteq$ $\bigoplus_{k_{1}, \cdots, k_{s} \geq 1} \mathbb{Z} / p\left\{x_{2 k_{1}-1} \otimes x_{2 k_{2}-1} \otimes \cdots \otimes x_{2 k_{s}-1}\right\} \subset H_{2 *+s}\left(\wedge^{s} P\right)$ and $\wedge^{s} P$ is a stable summand of $B V_{s}$. Now, any nontrivial element in $\left(S q^{0}\right)^{l} H_{*}\left(B V_{s}\right)$ may be written in the form

$$
x_{2 j_{1}-1} \otimes x_{2 j_{2}-1} \otimes \cdots \otimes x_{2 j_{s}-1}+\text { sum of monomials with higher order }
$$

with $2^{l}-1 \leq 2 j_{1}-1 \leq 2 j_{2}-1 \cdots \leq 2 j_{s}-1$, by changing the order of the factors if necessary. Therefore, by Proposition 2.1, it is enough to show

$$
\begin{aligned}
& u_{0}^{\left(2^{l-s}-1\right)}\left(x_{2 j_{1}-1} \otimes x_{2 j_{2}-1} \otimes \cdots \otimes x_{2 j_{s}-1}+\text { sum of monomials with higher order }\right) \\
& \neq 0 \in \bigoplus_{k_{1}, \cdots, k_{s} \geq 1} \mathbb{Z} / 2\left\{x_{2 k_{1}-1} \otimes x_{2 k_{2}-1} \otimes \cdots \otimes x_{2 k_{s}-1}\right\} \otimes \mathbb{Z} / 2\left[u_{s}\right] .
\end{aligned}
$$

But this is certainly the case, because Corollary 2.4 implies the left-hand side is

$$
\begin{array}{r}
u_{s}^{\left(2^{l-s}-1\right)}\left(x_{2\left(j_{1}-\left(2^{l-s}-1\right)\left(2^{s}-2^{s-1}\right)\right)-1} \otimes x_{2\left(j_{2}-\left(2^{l-s}-1\right)\left(2^{s-1}-2^{s-2}\right)\right)-1} \otimes \cdots\right. \\
\left.\otimes x_{2\left(j_{s}-\left(2^{l-s}-1\right)\right)-1}+\text { sum of monomials with higher order }\right),
\end{array}
$$

which is clearly nonzero, for

$$
\begin{aligned}
2\left(j_{1}-\left(2^{l-s}-1\right)\left(2^{s}-2^{s-1}\right)\right)-1 & \geq 2\left(2^{l-1}-\left(2^{l-s}-1\right)\left(2^{s}-2^{s-1}\right)\right)-1 \\
& =2^{s}-1 \geq 1
\end{aligned}
$$

and

$$
2 j_{1}-1 \leq 2 j_{2}-1 \leq \cdots \leq 2 j_{s}-1 .
$$

(ii) From Corollary 1.11 and Proposition 1.5,

$$
B P_{2 p^{l+s-1} t-s}\left(B V_{s}\right)=\left(\mathcal{P}^{0}\right)^{l}\left(P H_{2\left(p^{l+s-1} t-s\right)}\left(B T^{s}\right)\right) .
$$

Now, the rest of the proof goes as in (i).

\section{BP-ADAMS OPERATIONS ON $B P_{*}\left(\wedge^{s} P\right)$}

In this section, we study the action of the $B P$-Adams operation $\psi^{p+1}[\mathrm{~N}][\operatorname{Ad} 2]$ on $B P_{2 n-s}\left(\wedge^{s} P\right)$.

For our purpose, it is most convenient to establish a universal coefficient spectral sequence

$$
E_{2}=\operatorname{Ext}_{B P_{*}}^{*}\left(B P_{*}(X), B P_{*}\right) \Longrightarrow B P^{*}(X),
$$

using the chromatic tower [R3]. (For the original and different approach, see [Ad3].) Thus we now review materials related to the chromatic tower:

In their influential paper, Miller, Ravenel and Wilson [MRW] introduced the chromatic resolution

$$
B P_{*} \rightarrow M^{0} \rightarrow M^{1} \rightarrow M^{2} \rightarrow \cdots
$$

by splicing the short exact sequences

$$
0 \rightarrow N^{n} \rightarrow M^{n} \rightarrow N^{n+1} \rightarrow 0
$$

where $N^{0}=B P_{*}, M^{n}=v_{n}^{-1} N_{n}$. In addition to their usefulness for calculations [MRW], $M^{n}$ 's turned out to possess some nice properties. In fact, as Johnson, Landweber and Yoshimura [JLY] showed, each $M^{n}$ is $\mathcal{B P}$-injective, that is, $\operatorname{Ext}_{B P_{*}}^{i}\left(A, M^{n}\right)=0$ for all $i>0$ and all comodules $A$ in $\mathcal{B P}$, the category of 
all $B P_{*} B P$-comodules and comodule maps. Furthermore, using the technique of Bousfield [Bo], Ravenel [R3] showed both $M^{n}$ and $N^{n}$ are realizable as a spectrum: Let $L_{n}$ be the Bousfield localization functor [Bo] with respect to $v_{n}^{-1} B P$. Define inductively $N_{n}$ and $M_{n}$ so that $N_{0}=S^{0}, M_{n}=L_{n} N_{n}, N_{n+1}=$ the cofiber of the localization map $N_{n} \rightarrow L_{n} N_{n}=M_{n}$. Then it can be seen that $B P_{*}\left(N_{n}\right)=N^{n}, B P_{*}\left(M_{n}\right)=M^{n}$ [R3]. Thus the geometric chromatic resolution of the sphere looks like

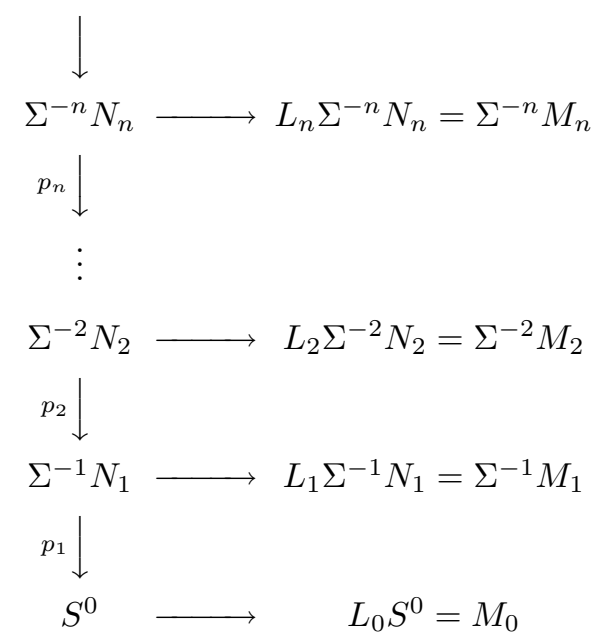

Here $p_{n}: \Sigma^{-n} N_{n} \rightarrow \Sigma^{-n+1} N_{n-1}$ is the fiber of the localization map $\Sigma^{-n+1} N_{n-1} \rightarrow$ $L_{n-1} \Sigma^{-n+1} N_{n-1}=\Sigma^{-n+1} M_{n-1}$.

Theorem 3.1. (i) Let $X$ be a countable union of finite spectra. Then there is a universal coefficient spectral sequence

$$
E_{2}^{s}=\operatorname{Ext}_{B P_{*}}^{s, *}\left(B P_{*}(X), B P_{*}\right) \Longrightarrow B P^{*}(X),
$$

such that $E_{1}^{s}=\operatorname{Hom}_{B P_{*}}^{*}\left(B P_{*}(X), M^{s}\right)$.

(ii) Let $Y$ be a finite spectrum. Then there is a universal coefficient spectral sequence

$$
E_{2}^{s}=\operatorname{Ext}_{B P_{*}}^{s, *}\left(B P^{*}(Y), B P_{*}\right) \Longrightarrow B P_{*}(Y)
$$

such that $E_{1}^{s}=\operatorname{Hom}_{B P_{*}}^{*}\left(B P^{*}(Y), M^{s}\right)$.

(iii) Suppose $Y=\operatorname{Colim} Y_{n}$ is a countable union of finite spectra. Then the same construction as in (ii) still gives us a spectral sequence converging to $B P_{*}(Y)$, such that $E_{1}^{s}=\operatorname{Hom}_{\text {cont }-B P_{*}}\left(B P^{*}(Y), M^{s}\right)$, where the topology of $B P^{*}(Y)$ is the inverse limit topology $B P^{*}(Y) \rightarrow \lim B P^{*}\left(Y_{n}\right)$. In other words,

$$
E_{1}^{s}=\lim _{\longrightarrow} \operatorname{Hom}_{B P_{*}}^{*}\left(B P^{*}\left(Y_{n}\right), M^{s}\right) .
$$

Hence the $E_{2}$-term becomes the homology of this continuous cochain complex, which we denote by $\operatorname{Ext}_{\text {cont-BP* }}^{\text {s,* }}\left(B P^{*}(Y), M^{s}\right)$, in analogy with the continuous cohomology. Thus we may write

$$
E_{2}^{s}=\operatorname{Ext}_{\text {cont-BP* }}^{s, *}\left(B P^{*}(Y), M^{s}\right)=\varliminf_{\longrightarrow} \operatorname{Ext}_{B P_{*}}^{s, *}\left(B P^{*}\left(Y_{n}\right), M^{s}\right) .
$$


Proof. (i) Let $f: X \rightarrow \Sigma^{*} B P \wedge S^{0}$ be a representative of an element of $B P^{*}(X)$. We introduce a decreasing filtration $B P^{*}(X)=F^{0} \supset F^{1} \supset \cdots$ so that $[f] \in F^{s} \Longleftrightarrow$ $f$ factorizes as

$$
X \rightarrow B P \wedge \Sigma^{-s} N_{s} \stackrel{B P \wedge\left(p_{1} \circ \cdots \circ p_{s}\right)}{\longrightarrow} B P \wedge S^{0}=B P .
$$

Clearly, $E_{1}^{s}=\left\{X, B P \wedge M_{s}\right\}^{*}$. We claim this is equal to $\operatorname{Hom}_{B P_{*}}^{*}\left(B P_{*}(X), M^{s}\right)$. In fact, this is the case for the spheres, since $B P_{*}\left(M_{s}\right)=M^{s}$. Then the claim for finite spectra is proved by the induction on the number of cells using the five lemma. The claim for countable unions of finite spectra is proved by noticing that the $\mathcal{B P}$-injectivity of $M^{n}$ implies the vanishing of $\lim ^{1}$ in the Milnor exact sequence.

Now, with this identification of the $E_{1}$-term, the claim about the $E_{2}$-term follows from the fact that the geometric differentials $d_{1}: B P \wedge M_{s} \rightarrow B P \wedge N_{s+1} \rightarrow$ $B P \wedge M_{s+1}$ realize the chromatic resolution $B P_{*} \rightarrow M^{0} \rightarrow M^{1} \rightarrow M^{2} \rightarrow \cdots$.

(ii): Let $g: S^{*} \rightarrow B P \wedge Y$ be a representative of an element of $B P_{*}(Y)$. We introduce a decreasing filtration $B P_{*}(Y)=F^{0} \supset F^{1} \supset \cdots$ so that $[g] \in F^{s} \Longleftrightarrow g$ factorizes as

$$
S^{*} \rightarrow B P \wedge \Sigma^{-s} N_{s} \wedge Y \stackrel{B P \wedge\left(p_{1} \circ \cdots \circ p_{s}\right) \wedge Y}{\longrightarrow} B P \wedge S^{0} \wedge Y=B P \wedge Y .
$$

Then the rest of the proof goes as in (i).

(iii) Having (ii), it is enough to identify the $E_{1}$-term. But this follows from the following commutative diagram:

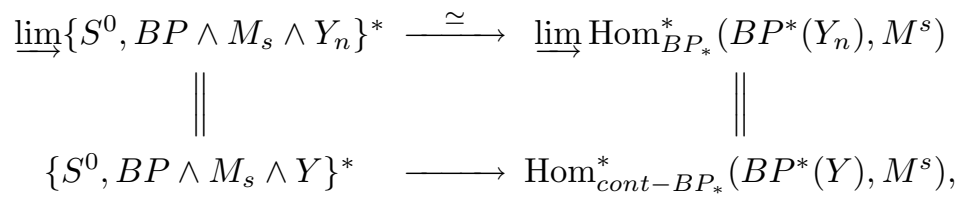

where all the maps are obvious ones.

The power of this theorem can be seen in the proof of the following:

Proposition 3.2. (i) For $Y=B V_{s}$, the filtration associated with spectral sequence of Theorem 3.1 (iii) looks like

$$
B P_{d}\left(B V_{s}\right)=F^{0}=F^{1} \supset F^{2} \supset \cdots \supset F^{s} \supset F^{s+1}=0
$$

and $F^{n}=F^{n+1}$ if $2 \nmid d+n$.

(ii) The above filtration is preserved by the action of $\psi^{p+1}$. Furthermore, when $2 \mid d+n, \psi^{p+1}$ acts on $E_{\infty}^{n, n+d}\left(B V_{s}\right)=F^{n} / F^{n+1}$ as multiplication by $(p+1)^{\frac{d+n}{2}}$.

(iii) Suppose $p^{\nu_{p}(m)}>\left[\frac{s-1}{2}\right]$. Then the $p$-exponent of any element in

is at most

$$
\left.\operatorname{Ker}\left(\psi^{p+1}-1\right)\right|_{B P_{2 m-s}\left(\wedge^{s} P\right)}
$$

$$
\begin{cases}1 & \text { if } p=2 \text { and } \nu_{2}(m)=0 \\ \nu_{2}(m)+2+\left[\frac{5\left[\frac{s-1}{2}\right]}{2}\right]-\alpha_{2}\left(\left[\frac{s-1}{2}\right]\right) & \text { if } p=2 \text { and } \nu_{2}(m) \geq 1 \\ \nu_{p}(m)+1+\frac{p\left[\frac{s-1}{2}\right]-\alpha_{p}\left(\left[\frac{s-1}{2}\right]\right)}{p-1} & \text { if } p \text { is odd. }\end{cases}
$$

Proof. (i) Clearly, $F^{0}=F^{1}$ and $F^{s}=F^{s+1}$, because $B P_{*}\left(B V_{s}\right)$ is $v_{0}$-torsion and $v_{s}$-local [JW]. Now, $F^{n} / F^{n+1}=E_{\infty}^{n, n+d}\left(B V_{s}\right)$ is a subquotient of $E_{1}^{n, n+d}\left(B V_{s}\right)=$ $\operatorname{Hom}_{B P_{*}}\left(B P^{2 *},\left(M^{n}\right)_{d+n-2 *}\right)$. But, this vanishes when $2 \nmid d+n$, as $\left(M^{n}\right)_{d+n-2 *}$ will then vanish. 
(ii) $\psi^{p+1}$ preserves the filtration because the filtration is associated with the geometric chromatic tower. For the second claim, it is enough to prove the same claim for $E_{1}^{n, n+d}\left(B V_{s}\right)=B P_{d}\left(B V_{s} \wedge \Sigma^{-n} M_{n}\right)$. Now we list some known facts about $\psi^{p+1}:$

(a) $\psi^{p+1}$ is a map of ring spectra.

(b) $\left.\psi^{p+1}\right|_{B P_{2 *}}$ is given as multiplication by $(p+1)^{*}$.

(c) For any $n,\left.\psi^{p+1}\right|_{B P_{2 *}\left(M_{n}\right)}$ is given as multiplication by $(p+1)^{*}$.

(d) $\left.\psi^{p+1}\right|_{B P^{2 *}\left(B V_{s}\right)}$ is given as multiplication by $(p+1)^{-*}$.

In fact, (a) and (b) are well-known and can be found in [Ar2][W]. (c) follows from (a) and (b), as $B P_{*}\left(M_{n}\right)=M^{n}$. Finally, (a), (b), and [L2] reduce (d) to the case $s=1$, when the claim is well-known $[\mathrm{H}]$.

To determine the $\psi^{p+1}$ action on $B P_{d}\left(B V_{s} \wedge \Sigma^{-n} M_{n}\right)$, our strategy is to embed it in $\operatorname{Hom}_{B P_{*}}^{*}\left(B P^{*}(Y), M^{s}\right)$ (as the submodule of all continuous $B P_{*}$ homomorphisms), and determine the $\psi^{p+1}$ action on $\operatorname{Hom}_{B P_{*}}^{*}\left(B P^{*}(Y), M^{s}\right)$ : Recall that the composite

$$
\begin{aligned}
\delta: B P_{d}\left(B V_{s} \wedge \Sigma^{-n} M_{n}\right) & =\operatorname{Hom}_{\text {cont }-B P_{*}}^{*}\left(B P^{2 *}\left(B V_{s}\right),\left(M^{n}\right)_{d+n-2 *}\right) \\
& \subseteq \operatorname{Hom}_{B P_{*}}^{*}\left(B P^{2 *}\left(B V_{s}\right),\left(M^{n}\right)_{d+n-2 *}\right)
\end{aligned}
$$

is defined by $\delta(x)(y)=\mu(x \otimes y)$, where $x \in B P_{d}\left(B V_{s} \wedge \Sigma^{-n} M_{n}\right), y \in B P^{2 *}\left(B V_{s}\right)$, and

$$
\mu: B P_{d}\left(B V_{s} \wedge \Sigma^{-n} M_{n}\right) \otimes B P^{2 *}\left(B V_{s}\right) \rightarrow\left(M^{n}\right)_{d+n-2 *}
$$

is the canonical pairing. Then, as $\psi^{p+1}$ is a map of ring spectra, we have the following commutative diagram:

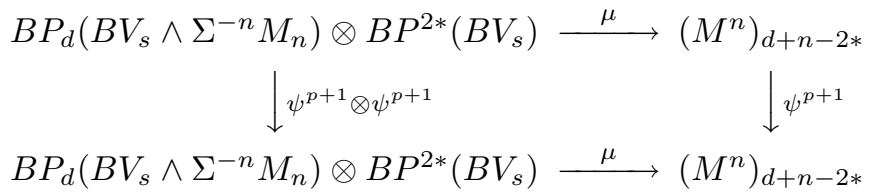

From this, we get the following commutative diagram

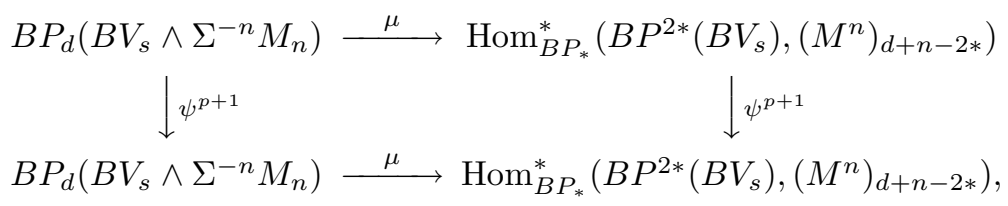

where $\psi^{p+1}$ acts on $\operatorname{Hom}_{B P_{*}}^{*}\left(B P^{2 *}\left(B V_{s}\right),\left(M^{n}\right)_{d+n-2 *}\right)$ by

$$
\left(\psi^{p+1} h\right)(y)=\psi^{p+1} h\left(\psi^{-(p+1)} y\right)
$$

for any $y \in B P^{2 *}\left(B V_{s}\right)$. But, as $\psi^{p+1} h\left(\psi^{-(p+1)} y\right)=(p+1)^{\frac{d+n}{2}-*} h\left((p+1)^{*} y\right)=$ $(p+1)^{\frac{d+n}{2}} h(y)$, we see $\psi^{p+1} h=(p+1)^{\frac{d+n}{2}} h$ from which the result follows. [M1]:

(iii) First, we recall the usual result in elementary number theory $[\mathrm{AA}][\mathrm{MM}][\mathrm{H}]$

$$
\nu_{p}\left((p+1)^{n}-1\right)=\nu_{p}(n)+\epsilon_{p}(n),
$$

where

$$
\epsilon_{p}(n)= \begin{cases}2 & \text { if } p=2 \text { and } \nu_{2}(n) \geq 1 \\ 1 & \text { otherwise }\end{cases}
$$


This is well-known and quite easy to prove: The key is to use mathematical induction to show

$$
\begin{aligned}
& (1+2)^{2^{r}} \equiv 1+2^{r+2} \quad\left(\bmod 2^{r+3}\right) \quad(r \geq 1), \\
& (1+p)^{p^{r}} \equiv 1+p^{r+1} \quad\left(\bmod p^{r+2}\right) \quad(p=\text { odd and } r \geq 0) .
\end{aligned}
$$

Consider the following procedure: Given $\left.z \in F^{n} \cap \operatorname{Ker}\left(\psi^{p+1}-1\right)\right|_{B P_{2 m-s}\left(\wedge^{s} P\right)}$, choose the following element in $\left.F^{n+1} \cap \operatorname{Ker}\left(\psi^{p+1}-1\right)\right|_{B P_{2 m-s}\left(\wedge^{s} P\right)}$ :

$$
\left\{\begin{array}{cl}
z & \text { if } 2 \nmid 2 m-s+n, \\
p^{\nu_{p}\left(\frac{2 m-s+n}{2}\right)+\epsilon_{p}\left(\frac{2 m-s+n}{2}\right)} z & \text { if } 2 \mid 2 m-s+n .
\end{array}\right.
$$

The validity of this procedure is guaranteed by (i) and (ii) of Proposition 3.2. Beginning with an arbitrary element

$$
\left.\omega \in \operatorname{Ker}\left(\psi^{p+1}-1\right)\right|_{B P_{2 m-s}\left(\wedge^{s} P\right)}=\left.F^{1} \cap \operatorname{Ker}\left(\psi^{p+1}-1\right)\right|_{B P_{2 m-s}\left(\wedge^{s} P\right)},
$$

we can repeat this procedure until we get

$$
\left.p^{e} \omega \in F^{s+1} \cap \operatorname{Ker}\left(\psi^{p+1}-1\right)\right|_{B P_{2 m-s}\left(\wedge^{s} P\right)}=0,
$$

where

$$
\begin{aligned}
e= & \left\{\nu_{p}\left(m-\left[\frac{s-1}{2}\right]\right)+\epsilon_{p}\left(m-\left[\frac{s-1}{2}\right]\right)\right\} \\
& +\left\{\nu_{p}\left(m-\left[\frac{s-1}{2}\right]+1\right)+\epsilon_{p}\left(m-\left[\frac{s-1}{2}\right]+1\right)\right\} \\
& +\cdots+\left\{\nu_{p}(m-1)+\epsilon_{p}(m-1)\right\} \\
& +\left\{\nu_{p}(m)+\epsilon_{p}(m)\right\} .
\end{aligned}
$$

But, as $p^{\nu_{p}(m)}>\left[\frac{s-1}{2}\right]$, this is equal to

$$
\begin{aligned}
= & \left\{\nu_{p}(m)+\epsilon_{p}(m)\right\}+\nu_{p}\left(\left[\frac{s-1}{2}\right] !\right) \\
& +\epsilon_{p}(1)+\epsilon_{p}(2)+\cdots+\epsilon_{p}\left(\left[\frac{s-1}{2}\right]\right) \\
= & \begin{cases}1 & \text { if } p=2 \text { and } \nu_{2}(m)=0, \\
\nu_{2}(m)+2+\left(\left[\frac{s-1}{2}\right]-\alpha_{2}\left(\left[\frac{s-1}{2}\right]\right)\right)+\left[\frac{3\left[\frac{s-1}{2}\right]}{2}\right] & \text { if } p=2 \text { and } \nu_{2}(m) \geq 1, \\
\nu_{p}(m)+1+\frac{\left[\frac{s-1}{2}\right]-\alpha_{p}\left(\left[\frac{s-1}{2}\right]\right)}{p-1}+\left[\frac{s-1}{2}\right] & \text { if } p \text { is odd }\end{cases} \\
= & \begin{array}{ll}
1 & \text { if } p=2 \text { and } \nu_{2}(m)=0, \\
\nu_{2}(m)+2+\left[\frac{5\left[\frac{s-1}{2}\right]}{2}\right]-\alpha_{2}\left(\left[\frac{s-1}{2}\right]\right) & \text { if } p=2 \text { and } \nu_{2}(m) \geq 1, \\
\nu_{p}(m)+1+\frac{p\left[\frac{s-1}{2}\right]-\alpha_{p}\left(\left[\frac{s-1}{2}\right]\right)}{p-1} & \text { if } p \text { is odd }
\end{array}
\end{aligned}
$$

where we used the well-known result $\nu_{p}(n !)=\frac{n-\alpha_{p}(n)}{p-1}$ in the third equality. Now the proof is complete. 


$$
\text { 4. The MAP } P H_{n}\left(B V_{s}\right) \rightarrow \operatorname{Ext}_{\mathcal{A}_{*}}^{s, n+s}(\mathbb{Z} / p, \mathbb{Z} / p)
$$

In this section, we study the key transfer homomorphism

$$
P H_{n}\left(B V_{s}\right) \rightarrow \operatorname{Ext}_{\mathcal{A}_{*}}^{s, n+s}(\mathbb{Z} / p, \mathbb{Z} / p)
$$

in detail. This was first systematically studied by Singer [S1] (see $\S 6$ for more about this). Our principal goal here is Theorem 4.4, where we show $S q^{0}$ 's (resp. $\mathcal{P}^{0}$ 's) on $P H_{*}\left(B V_{s}\right)$ and $\operatorname{Ext}_{\mathcal{A}_{*}}^{s, s+*}(\mathbb{Z} / p, \mathbb{Z} / p)$ commute when $p=2$ (resp. $p$ is odd). This was shown in [B] for $s \leq 3$ when $p=2$. We primarily consider the odd primary case, because the case $p=2$ is similar (and simpler). Our presentation here is strongly influenced by $[\mathrm{B}]$, which treated the case $p=2$.

First, we give an explicit set of generators for $H^{*}(B \mathbb{Z} / p)$, using the generator $c \in H^{*}\left(\mathbb{C} P^{\infty}\right) \cong \mathbb{Z} / p[c]$. Consider the $S^{1}$-bundle $S^{1} \rightarrow B \mathbb{Z} / p \rightarrow \mathbb{C} P^{\infty}$. From this, we get the Gysin sequence

$$
\cdots \stackrel{p^{*}}{\rightarrow} H^{i+1}(B \mathbb{Z} / p) \stackrel{\tau}{\rightarrow} H^{i}\left(\mathbb{C} P_{+}^{\infty}\right) \stackrel{\chi_{\dot{*}}}{\rightarrow} H^{i+2}\left(\mathbb{C} P^{\infty}\right) \stackrel{p^{*}}{\rightarrow} H^{i+2}\left(B V_{1}\right) \stackrel{\tau}{\rightarrow} \cdots
$$

where $\tau$ is induced by the $S^{1}$-transfer $\Sigma \mathbb{C} P_{+}^{\infty} \rightarrow B V_{1}$ and $\chi \cdot$ is multiplication by the Euler class $p c \in H^{2}\left(\mathbb{C} P^{\infty}\right)$, which is of course 0 when we consider the mod $p$ cohomology. Define $a \in H^{1}(B \mathbb{Z} / p)$ and $b \in H^{2}(B \mathbb{Z} / p)$ so that $\tau(a)=1 \in H^{0}\left(\mathbb{C} P_{+}^{\infty}\right)$ and $p^{*}(c)=b \in H^{2}(B \mathbb{Z} / p)$. Since $\tau$ is a $H^{*}\left(\mathbb{C} P^{\infty}\right)$-module homomorphism by the projection formula, $\tau\left(a b^{k}\right)=c^{k}$, which in turn implies $H^{*}(B \mathbb{Z} / p) \cong E[a] \otimes \mathbb{Z} / p[b]$.

We now claim $\beta(a)=b$, where $\beta$ is the Bockstein homomorphism. For this purpose, we recall we may derive the Gysin sequence by applying the Leray-Serre spectral sequence

$$
E_{2}^{p, q}=H^{p}\left(\mathbb{C} P^{\infty}, \mathbb{Z}\right) \otimes H^{q}\left(S^{1}\right) \Longrightarrow H^{p+q}(B \mathbb{Z} / p) .
$$

In the Leray-Serre $E_{2}$-term, we may write $a=1 \otimes g$ and $b=\tilde{c} \otimes 1$, where $g \in H^{1}\left(S^{1}\right)$ is the generator and $\tilde{c}$ is the generator of $H^{2}\left(\mathbb{C} P^{\infty}, \mathbb{Z}\right)$ which lifts $c$. Our plan is to compare the above Leray-Serre spectral sequence with its mod $p^{2}$ cohomology version

$$
\tilde{E}_{2}^{p, q}=H^{p}\left(\mathbb{C} P^{\infty}, \mathbb{Z}\right) \otimes H^{q}\left(S^{1}, \mathbb{Z} / p^{2}\right) \Longrightarrow H^{p+q}\left(B \mathbb{Z} / p, \mathbb{Z} / p^{2}\right),
$$

via the $\bmod p$ reduction, following the definition of $\beta$. So, we first lift $1 \otimes g$ to $1 \otimes \tilde{g}$, where $\tilde{g} \in H^{1}\left(S^{1}, \mathbb{Z} / p^{2}\right)$ is a generator which lifts $g$. Then, we must apply the coboundary map (in the cochain complex for the Leray-Serre spectral sequence) to $1 \otimes \tilde{g}$. However, since this cobounday map induces $d_{2}$ in the Leray-Serre spectral sequence, it suffices to evaluate

$$
d_{2}(1 \otimes \tilde{g})=p \tilde{c} \otimes 1 .
$$

Thus, $\beta(a)$ is detected in the Leray-Serre $E_{2}$-term by the $\bmod p$ reduction of $\tilde{c} \otimes 1$. This proves $\beta(a)=b$.

Now, we define $x_{i} \in H_{i}(B \mathbb{Z} / p)$ as the dual of $a^{e} b^{n} \in H^{i}(B \mathbb{Z} / p)$, where $i=e+n$ with $e=0$ or 1 . Since $\tau\left(a b^{k}\right)=c^{k}$, this definition coincides with the one which was listed in Notation and Convention in $\S 0$.

Next, we define an $\mathcal{A}^{*}$-module $T$ by

$$
T=\mathbb{Z} / p\left\{a^{e} b^{n} \mid e=0,1 \text { and } n \geq 0, \text { or } e=1 \text { and } n \geq-1\right\}
$$


so that

$$
\begin{gathered}
\mathcal{P}\left(a b^{-1}\right)=a\left(b+b^{p}\right)^{-1}=a b^{-1}\left(1+b^{p-1}\right)^{-1}, \\
\beta\left(a b^{-1}\right)=1 .
\end{gathered}
$$

In fact $T=H^{*}(R)$, where $R$ is the desuspension of the cofiber of the Kahn-Priddy map $B \mathbb{Z} / p_{+} \rightarrow S^{0}$. Now the following morphism of short exact sequences of $\mathcal{A}^{*}$ modules is essential to our approach:

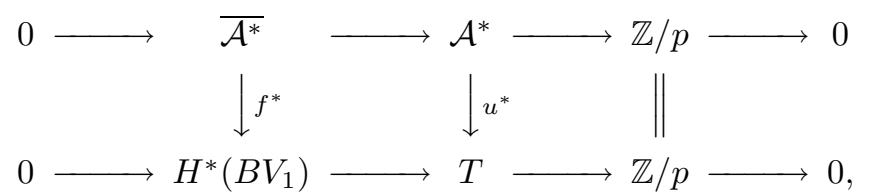

where unnamed maps are obvious ones, $u^{*}$ is an $\mathcal{A}^{*}$-module map of degree -1 characterized by $u^{*}(1)=a b^{-1}$, and $f^{*}$ is the restriction of $u^{*}$. It is well-known and easy to see $[\mathrm{B}]$ that this is induced from a diagram of spectra maps

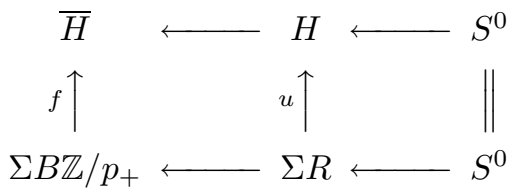

Applying $-\otimes\left(f^{*}\right)^{\otimes i}$ to this, we get another morphism of short exact sequences of $\mathcal{A}^{*}$-modules:

$\left(C_{i}\right)$

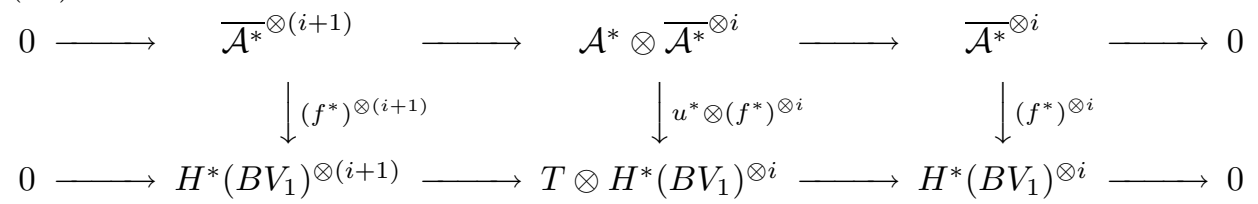

Of course, we may simply define

$$
P H_{*}\left(B V_{s}\right) \cong \operatorname{Hom}_{\mathcal{A}^{*}}\left(H^{*}\left(B V_{1}\right)^{\otimes s}, \mathbb{Z} / p\right) \rightarrow \operatorname{Ext}_{\mathcal{A}^{*}}^{s}(\mathbb{Z} / p, \mathbb{Z} / p)
$$

as the composition of $s$ connecting homomorphisms associated with the lower short exact sequence of $\left(C_{i}\right)$ for $0 \leq i \leq s-1$. However, for our purpose, we should write down this map explicitly. Therefore, we have to make full use of $\left(C_{i}\right)$ :

Notice that we can get a free $\mathcal{A}^{*}$-resolution of $\mathbb{Z} / p$

$$
\cdots \rightarrow \mathcal{A}^{*} \otimes{\overline{\mathcal{A}^{*}}}^{\otimes i} \rightarrow \mathcal{A}^{*} \otimes{\overline{\mathcal{A}^{*}}}^{\otimes(i-1)} \rightarrow \cdots \mathcal{A}^{*} \rightarrow \mathbb{Z} / p,
$$

by splicing the upper short exact sequences of $\left(C_{i}\right)$ 's. Using this, we can calculate $\operatorname{Ext}_{\mathcal{A}^{*}}^{*}(\mathbb{Z} / p, \mathbb{Z} / p)$ as the homology of the cochain complex

$$
\begin{aligned}
0 & \rightarrow \mathbb{Z} / p=\operatorname{Hom}_{\mathcal{A}^{*}}\left(\mathcal{A}^{*}, \mathbb{Z} / p\right) \rightarrow \operatorname{Hom}_{\mathcal{A}^{*}}\left(\mathcal{A}^{*} \otimes \overline{\mathcal{A}^{*}}, \mathbb{Z} / p\right) \cdots \\
& \rightarrow \operatorname{Hom}_{\mathcal{A}^{*}}\left(\mathcal{A}^{*} \otimes{\overline{\mathcal{A}^{*}}}^{\otimes(i-1)}, \mathbb{Z} / p\right) \rightarrow \operatorname{Hom}_{\mathcal{A}^{*}}\left(\mathcal{A}^{*} \otimes{\overline{\mathcal{A}^{*}}}^{\otimes i}, \mathbb{Z} / p\right) \cdots .
\end{aligned}
$$

Given an $\mathcal{A}^{*}$-module $M$, define ${ }_{f} M$ to be $M$ equipped with the trivial $\mathcal{A}^{*}$-module structure. Then we have the well-known isomorphism

$$
\operatorname{Hom}_{\mathcal{A}^{*}}\left(\mathcal{A}^{*} \otimes M, \mathbb{Z} / p\right) \stackrel{\phi^{*}}{\cong} \operatorname{Hom}_{\mathcal{A}^{*}}\left(\mathcal{A}^{*} \otimes{ }_{f} M, \mathbb{Z} / p\right) \cong \operatorname{Hom}_{\mathbb{Z} / p}(M, \mathbb{Z} / p)=M^{*}
$$


Here $\phi: \mathcal{A}^{*} \otimes{ }_{f} M \rightarrow \mathcal{A}^{*} \otimes M$ is an $\mathcal{A}^{*}$-module isomorphism given by

$$
\phi: \mathcal{A}^{*} \otimes{ }_{f} M \stackrel{\Delta \otimes M}{\longrightarrow} \mathcal{A}^{*} \otimes \mathcal{A}^{*} \otimes_{f} M \stackrel{A \otimes \epsilon \alpha}{\longrightarrow} \mathcal{A}^{*} \otimes \mathbb{Z} / p \otimes M=\mathcal{A}^{*} \otimes M,
$$

where $\Delta: \mathcal{A}^{*} \rightarrow \mathcal{A}^{*} \otimes \mathcal{A}^{*}$ is the coproduct and $\epsilon: \mathcal{A}^{*} \rightarrow \mathbb{Z} / p$ is the augmentation. Thus, we can calculate $\operatorname{Ext}_{\mathcal{A}^{*}}^{*}(\mathbb{Z} / p, \mathbb{Z} / p)$ as the homology of the cochain complex

$$
0 \rightarrow \mathbb{Z} / p \rightarrow \overline{\mathcal{A}_{*}} \rightarrow \cdots \overline{\mathcal{A}}_{*}^{\otimes(i-1)} \rightarrow{\overline{\mathcal{A}_{*}}}^{\otimes i} \rightarrow \cdots
$$

where we used the obvious isomorphism $\left(\mathcal{A}^{*}\right)^{*} \cong \mathcal{A}_{*}$. Of course, this construction applies equally to general augmented Hopf algebras, and is functorial with respect to maps between augmented Hopf algebras. However, this is not the usual normalized inhomogeneous bar resolution, though we can construct explicit isomorphisms between these two resolutions via $\phi$ and its inverse. In fact, this is the normalized homogeneous bar resolution ([AD] called (normalized) Hopf bar resolution). But, all we need here is that this resolution is functorial.

We are now ready to give a chain-level description of the key transfer homomorphism

$$
P H_{*}\left(B V_{s}\right) \cong \operatorname{Ext}_{\mathcal{A}^{*}}^{0}\left(H^{*}\left(B V_{1}\right)^{\otimes s}, \mathbb{Z} / p\right) \rightarrow \operatorname{Ext}_{\mathcal{A}^{*}}^{s}(\mathbb{Z} / p, \mathbb{Z} / p)
$$

Lemma 4.1. (i) The identification $P H_{*}\left(B V_{s}\right) \cong \operatorname{Hom}_{\mathcal{A}^{*}}\left(H^{*}\left(B V_{1}\right)^{\otimes s}, \mathbb{Z} / p\right)$ is given by the composite

$$
\begin{aligned}
\operatorname{Hom}_{\mathcal{A}^{*}}\left(H^{*}\left(B V_{1}\right)^{\otimes s}, \mathbb{Z} / p\right) & \subset \operatorname{Hom}_{\mathcal{A}^{*}}\left(\mathcal{A}^{*} \otimes H^{*}\left(B V_{1}\right)^{\otimes s}, \mathbb{Z} / p\right) \\
& \stackrel{\phi^{*}}{\cong} \operatorname{Hom}_{\mathcal{A}^{*}}\left(\mathcal{A}^{*} \otimes{ }_{f} H^{*}\left(B V_{1}\right)^{\otimes s}, \mathbb{Z} / p\right) \\
& \cong \operatorname{Hom}_{\mathbb{Z} / p}\left(H^{*}\left(B V_{1}\right)^{\otimes s}, \mathbb{Z} / p\right)=H_{*}\left(B V_{s}\right)
\end{aligned}
$$

where the first map (inclusion) is induced by the surjective map $\epsilon \otimes 1: \mathcal{A}^{*} \otimes$ $H^{*}\left(B V_{1}\right)^{\otimes s} \rightarrow H^{*}\left(B V_{1}\right)^{\otimes s}$.

(ii) The map $P H_{*}\left(B V_{s}\right) \rightarrow \operatorname{Ext}_{\mathcal{A}^{*}}^{s}(\mathbb{Z} / p, \mathbb{Z} / p)$ is induced by

$$
H_{*}\left(B V_{1}\right)^{\otimes s} \stackrel{\left(f_{*}\right)^{\otimes s}}{\longrightarrow} \overline{\mathcal{A}^{*}}{ }^{\otimes s},
$$

where $\overline{\mathcal{A}^{* \otimes s}}$ is regarded as the $s$-th module in the normalized homogeneous bar complex (Hopf bar complex) to calculate Ext over $\mathcal{A}^{*}$.

Proof. (i) is clear. (ii) follows from the following commutative diagram:

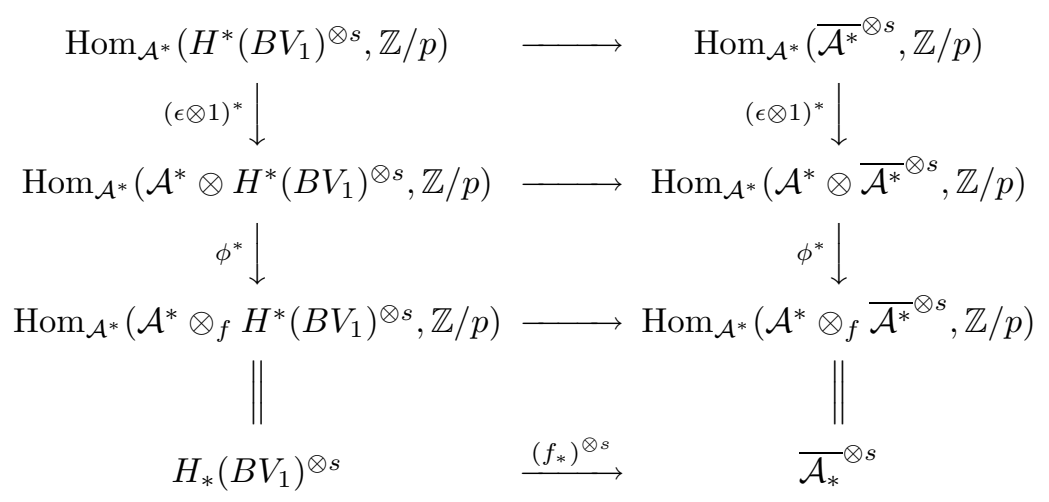


Now we are naturally led to study $f_{*}$. As was noted in $[\mathrm{B}]$, the determination of $f_{*}$ is reduced to a study of Milnor's $[\mathrm{Mi}] \lambda^{*}$ homomorphism (i.e. right $\mathcal{A}_{*}$-coaction):

$$
\lambda^{*}: H^{*}(X) \rightarrow H^{*}(X) \widehat{\otimes} \mathcal{A}_{*} .
$$

We choose the generator $x_{i} \in H_{i}\left(B V_{1}\right)$ so that it is dual to $a^{e} b^{n} \in H^{i}\left(B V_{1}\right)$ with $\left|a^{e} b^{n}\right|=i$, as was done at the beginning of this section. Then we have

\section{Lemma 4.2.}

$f_{*}\left(x_{i}\right)=(-1) \cdot$ the coefficient of $a^{e} b^{n}$ with $\left|a^{e} b^{n}\right|=i$ in $\lambda^{*}\left(a b^{-1}\right) \in H^{*}(R) \widehat{\otimes} \mathcal{A}_{*}$.

Proof. For simplicity, we set

$$
\begin{gathered}
A=\text { the coefficient of } a^{e} b^{n} \text { with }\left|a^{e} b^{n}\right|=i \\
\text { in } \lambda^{*}\left(a b^{-1}\right) \in H^{*}(R) \widehat{\otimes} \mathcal{A}_{*}, \\
B=\text { the coefficient of } x_{-1} \text { in } \eta_{*}\left(x_{i}\right),
\end{gathered}
$$

where $\eta_{*}: H_{*}(X) \rightarrow \mathcal{A}_{*} \otimes H_{*}(x)$ is the left $\mathcal{A}_{*}$-coaction. Notice that both $A$ and $B$ belong to $\mathcal{A}_{i+1}$. First, we claim that

$$
f_{*}\left(x_{i}\right)=(-1)^{i+1} B .
$$

In fact, this follows from the following commutative diagram:

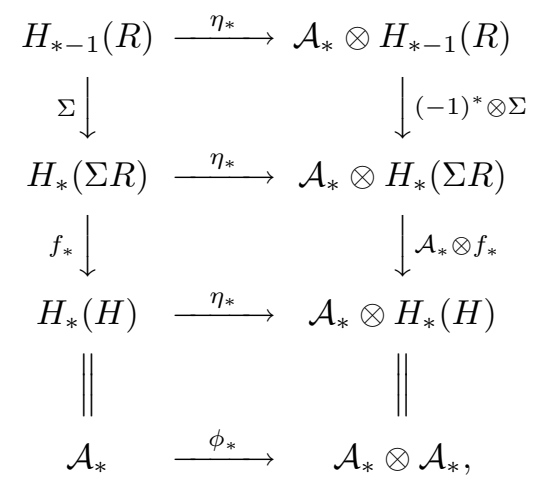

where $\phi_{*}$ is the coproduct of $\mathcal{A}_{*}$.

Now, for any $y \in \mathcal{A}^{i}$, we have

$$
\begin{aligned}
\left\langle\eta_{*}\left(x_{i}\right), y \otimes a b^{-1}\right\rangle & =\left\langle x_{i}, y \cdot\left(a b^{-1}\right)\right\rangle=\left\langle x_{i} \cdot y, a b^{-1}\right\rangle \\
& =\left\langle\lambda_{*}\left(x_{i} \otimes y\right), a b^{-1}\right\rangle=\left\langle x_{i} \otimes y, \lambda^{*}\left(a b^{-1}\right\rangle .\right.
\end{aligned}
$$

But, as

$$
\begin{aligned}
\left\langle\eta_{*}\left(x_{i}\right), y \otimes a b^{-1}\right\rangle & =\left\langle B \otimes x_{-1}, y \otimes\left(a b^{-1}\right)\right\rangle \\
& =(-1)^{(-1) i}\langle B, y\rangle\left\langle x_{-1} \otimes\left(a b^{-1}\right)\right\rangle=(-1)^{i}\langle B, y\rangle
\end{aligned}
$$

and

$$
\begin{aligned}
\left\langle x_{i} \otimes y, \lambda^{*}\left(a b^{-1}\right)\right\rangle & =\left\langle x_{i} \otimes y, a^{e} b^{n}\right\rangle \\
& =(-1)^{i \cdot i}\left\langle x_{i}, a^{e} b^{n}\right\rangle\langle y, A\rangle \\
& =(-1)^{i \cdot i+i \cdot i}\langle A . y\rangle=\langle A, y\rangle,
\end{aligned}
$$

we have

$$
B=(-1)^{i} A .
$$


Therefore, we get

$$
f_{*}\left(x_{i}\right)=(-1)^{i+1} B=-A,
$$

which completes the proof.

To calculate $\lambda^{*}\left(a b^{-1}\right)$, recall

$$
\begin{aligned}
& \lambda^{*}(a)=a \otimes 1+b \otimes \tau_{0}+b^{p} \otimes \tau_{1}+\cdots+b^{p^{r}} \otimes \tau_{r}+\cdots, \\
& \lambda^{*}(b)=b \otimes 1+b^{p} \otimes \xi_{1}+\cdots+b^{p^{r}} \otimes \xi_{r}+\cdots .
\end{aligned}
$$

In fact, these are nothing but the definitions of $\tau_{i}$ 's and $\xi_{j}$ 's [Mi]. Then, using the $p$-adic convergent expansion $-1=\sum_{j \geq 0}(p-1) p^{j}$, we have

$$
\begin{aligned}
\lambda^{*}\left(a b^{-1}\right)= & \left(a \otimes 1+b \otimes \tau_{0}+b^{p} \otimes \tau_{1}+\cdots+b^{p^{r}} \otimes \tau_{r}+\cdots\right) \\
& \cdot\left(b \otimes 1+b^{p} \otimes \xi_{1}+\cdots+b^{p^{r}} \otimes \xi_{r}+\cdots\right)^{-1} \\
= & \left(a \otimes 1+b \otimes \tau_{0}+b^{p} \otimes \tau_{1}+\cdots+b^{p^{r}} \otimes \tau_{r}+\cdots\right) \\
& \cdot\left(b^{-1} \otimes 1\right)\left(1 \otimes 1+b^{p-1} \otimes \xi_{1}+\cdots+b^{p^{r}-1} \otimes \xi_{r}+\cdots\right)^{-1} \\
= & \left(a b^{-1} \otimes 1+1 \otimes \tau_{0}+b^{p-1} \otimes \tau_{1}+\cdots+b^{p^{r}-1} \otimes \tau_{r}+\cdots\right) \\
& \cdot \prod_{j \geq 0}\left(1 \otimes 1+b^{(p-1) p^{j}} \otimes \xi_{1}^{p^{j}}+\cdots+b^{\left(p^{r}-1\right) p^{j}} \otimes \xi_{r}^{p^{j}}+\cdots\right)^{p-1}
\end{aligned}
$$

Lemma 4.3. Let $F: \mathcal{A}_{*} \rightarrow \mathcal{A}_{*}$ be the Frobenius homomorphism given by $x \mapsto x^{p}$. Then

(i) $F f_{*}\left(x_{2 i}\right)=0$,

(ii) $f_{*}\left(x_{2 i-1}\right)=0$ if $p-1 \nmid i$,

(iii) $f_{*}\left(\mathcal{P}^{0} x_{n}\right)=F f_{*}\left(x_{n}\right)$.

Proof. (i) This is because all the coefficients of the terms of the expansion of $b^{i}$ involve some $\tau_{j}$ whose square vanishes.

(ii) Just notice that $a b^{-1} b^{i}$ does not show up in the expansion of $\lambda^{*}\left(a b^{-1}\right)$ as far as $p-1 \nmid i$.

(iii) From (i), we may assume $n=2 k-1$. We must show $f_{*}\left(x_{2 k p-1}\right)=f_{*}\left(x_{2 k-1}\right)^{p}$. For this, notice that the coefficient of $a b^{-1} b^{k p}$ in $\lambda^{*}\left(a b^{-1}\right)$ is the same as that of $b^{k p}$ in

$$
\prod_{j \geq 0}\left(1 \otimes 1+b^{(p-1) p^{j}} \otimes \xi_{1}^{p^{j}}+\cdots+b^{\left(p^{r}-1\right) p^{j}} \otimes \xi_{r}^{p^{j}}+\cdots\right)^{p-1}
$$

But the only way to obtain $b^{k p}$ from here is to use the term $1 \otimes 1$ from the factor $j=0$, while the other factors become

$$
\begin{aligned}
& \prod_{j \geq 1}\left(1 \otimes 1+b^{(p-1) p^{j}} \otimes \xi_{1}^{p^{j}}+\cdots+b^{\left(p^{r}-1\right) p^{j}} \otimes \xi_{r}^{p^{j}}+\cdots\right)^{p-1} \\
= & \left(\prod_{j \geq 0}\left(1 \otimes 1+b^{(p-1) p^{j}} \otimes \xi_{1}^{p^{j}}+\cdots+b^{\left(p^{r}-1\right) p^{j}} \otimes \xi_{r}^{p^{j}}+\cdots\right)^{p-1}\right)^{p} .
\end{aligned}
$$

Therefore the claim follows.

We have two $S q^{0}$ 's (resp. $\mathcal{P}^{0}$ 's), one for $P H_{*}\left(B V_{s}\right)$ defined in Corollary 1.10 (ii) (resp. at the end of $\S 1$ ), and another for $\operatorname{Ext}_{\mathcal{A}_{*}^{*}}^{s,}(\mathbb{Z} / p, \mathbb{Z} / p)$, for $p=2$ (resp. $p$ odd). Now the following is our main result in this section. 
Theorem 4.4. The transfer homomorphism commutes with the two $S q^{0}$ 's (resp. $\mathcal{P}^{0}$ 's) for $p=2$ (resp. $p$ odd).

Proof. We prove this only for $p$ odd. (The case $p=2$ is similar.) Let $V: \mathcal{A}^{*} \rightarrow$ $\mathcal{A}^{*}$ be the dual of the Frobenius $F: \mathcal{A}_{*} \rightarrow \mathcal{A}_{*}$. Then, as $V$ is a morphism of cocommutative Hopf algebra over $\mathbb{Z} / p$, Lemma 4.1 and Lemma 4.3 imply that the following diagram commutes:

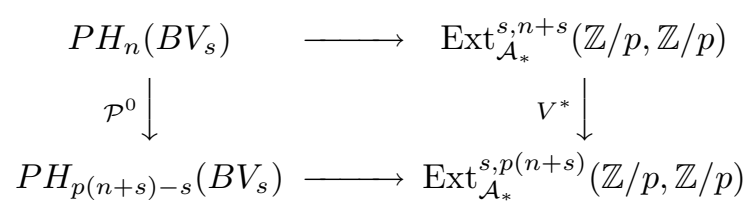

But, as is well-known (cf. [My]),

$$
V^{*}=\mathcal{P}^{0}: \operatorname{Ext}_{\mathcal{A}_{*}}^{s, n+s}(\mathbb{Z} / p, \mathbb{Z} / p) \rightarrow \operatorname{Ext}_{\mathcal{A}_{*}}^{s, p(n+s)}(\mathbb{Z} / p, \mathbb{Z} / p)
$$

with the convention $\mathcal{P}^{0}=0$ if $2 \nmid n+s$. (Notice that $V^{*}=0$ when $2 \nmid n+s$.) This completes the proof.

\section{THE MAIN THEOREM AND ITS PROOF}

Now we are ready to state and prove our main theorem.

Theorem 5.1. The Thom reduction of the primitive parts

$$
P B P_{2 n-s}\left(B V_{s}\right) \rightarrow P H_{2 n-s}\left(B V_{s}\right)
$$

is a trivial map, when

$$
\left\{\begin{array}{c}
2^{\nu_{2}(n)+2-2 s}>\nu_{2}(n)+2+\left[\frac{5\left[\frac{s-1}{2}\right]}{2}\right]-\alpha_{2}\left(\left[\frac{s-1}{2}\right]\right) \quad \text { if } p=2, \\
p^{\nu_{p}(n)+2-2 s}>(p-1) \nu_{p}(n)+1+p\left[\frac{s-1}{2}\right]-\alpha_{p}\left(\left[\frac{s-1}{2}\right]\right) \quad \text { if } p \text { is odd } .
\end{array}\right.
$$

Proof. Notice that both

$$
p^{\nu_{p}(n)}>\left[\frac{s-1}{2}\right] \quad \text { for any } p
$$

and

$$
\nu_{p}(n) \geq\left[\log _{p}\left[\frac{s}{2}\right]\right]+s-1 \quad \text { for } p \text { odd and } s \geq 2
$$

hold under the assumption. Hence we may apply both Proposition 3.2(iii) and Proposition 2.5. Now the claim is an immediate consequence of these two propositions.

Corollary 5.2. Under the same assumption as Theorem 5.1, the stable Hurewicz map

$$
\pi_{2 n-s}^{s}\left(B V_{s}\right) \rightarrow H_{2 n-s}\left(B V_{s}\right)
$$

has a trivial image.

Now, let us recall the new doomsday conjecture proposed in [M3]: 
New doomsday conjecture. For each $s$, there exists some integer $n(s)$ such that no nontrivial element in the image of

$$
\left(\mathcal{P}^{0}\right)^{n(s)}\left(\operatorname{Ext}_{\mathcal{A}_{*}}^{s, *}(\mathbb{Z} / p, \mathbb{Z} / p)\right) \subseteq\left(\operatorname{Ext}_{\mathcal{A}_{*}}^{s, p^{n(s)} *}(\mathbb{Z} / p, \mathbb{Z} / p)\right)
$$

is a nontrivial permanent cycle.

Hence, Corollary 5.2 immediately implies

Corollary 5.3. The iterated transfer analogue of the new doomsday conjecture holds: For each $s$, there exists some integer $n(s)$ such that no nontrivial element in the image of

$$
\left(\mathcal{P}^{0}\right)^{n(s)}\left(\operatorname{Ext}_{\mathcal{\mathcal { A }}_{*}^{*}}^{s, *}(\mathbb{Z} / p, \mathbb{Z} / p)\right) \subseteq\left(\operatorname{Ext}_{\mathcal{A}_{*}}^{s, p^{n(s)} *}(\mathbb{Z} / p, \mathbb{Z} / p)\right)
$$

comes from $\pi_{p^{n(s) *-s}}^{s}\left(B V_{s+}\right)$ via the s-fold iterated transfer.

\section{Afterthoughts}

To conclude, let us discuss a possible way to get around Singer's counterexample [S1]. Singer [S1] showed the iterated transfers actually induce a map of graded rings

$$
\bigoplus_{s} P H_{*}\left(B(\mathbb{Z} / p)^{s}\right) / G L_{s}(\mathbb{Z} / p) \rightarrow \bigoplus_{s} \operatorname{Ext}_{\mathcal{A}_{*}^{s+s}}^{s,+}(\mathbb{Z} / p, \mathbb{Z} / p),
$$

but he found this is not surjective when $p=2, *=9$, and $s=5$.

Now, from the new doomsday philosophy, especially by the way in which we used Kameko's reduction in the proof of Theorem 5.1, it appears that the right map to consider is the following localized version of Singer's homomorphism:

$$
\bigoplus_{s}\left(\mathcal{P}^{0}\right)^{-1} P H_{*}\left(B(\mathbb{Z} / p)^{s}\right) / G L_{s}(\mathbb{Z} / p) \rightarrow \bigoplus_{s}\left(\mathcal{P}^{0}\right)^{-1} \operatorname{Ext}_{\mathcal{A}_{*}^{s *+s}}^{*^{*+s}}(\mathbb{Z} / p, \mathbb{Z} / p) .
$$

If this localized Singer homomorphism were shown to be an isomorphism, then our Theorem 5.1 may be regarded as a strong supporting evidence of the new doomsday conjecture.

\section{REFERENCES}

[Ad1] J. F. Adams, On the non-existence of elements of Hopf invariant one, Ann. of Math. 72 (1960), 20-104. MR 25:4530

[Ad2] J. F. Adams, Vector fields on spheres, Ann. of Math. 75 (1962), 603-632. MR 25:2614

[Ad3] J. F. Adams, Lectures on Generalised Cohomology, Lecture Notes in Mathematics, vol. 99, Springer-Verlag, 1969, pp. 1-138. MR 40:4943

[AA] J. F. Adams and M. F. Atiyah, K-theory and the Hopf invariant, Quart. J. Math. Oxford 17 (1966), 31-38. MR 33:6618

[AGM] J. F. Adams, J. H. Gunawardena and H. R. Miller, The Segal conjecture for elementary abelian p-groups, Topology 24 (1985), 435-460. MR 87m:55026

[AD] D. W. Anderson and D. M. Davis, A vanishing theorem in homological algebra, Comm. Math. Helv. 48 (1973), 318-327. MR 48:12526

[Ar1] S. Araki, Typical Formal Groups in Complex Cobordism and K-Theory, Lecture Notes Math., Kyoto Univ. 6, Kinokuniya Book Store, 1973. MR 51:11549

[Ar2] S. Araki, Multiplicative operations in BP cohomology, Osaka J. Math. 12 (1975), 343-356.

[B] J. M. Boardman, Modular representations on the homology of powers of real projective space, Algebraic Topology; Oaxtepec 1991 (M. C. Tangora, ed.), Contemp. Math., vol. 146, American Mathematical Society, 1993, pp. 49-70. MR 95a:55041

[Bo] A. K. Bousfield, The localization of spectra with respect to homology, Topology 18 (1979), 257-281. MR 80m:55006 
[C1] G. Carlsson, G. B. Segal's Burnside ring conjecture for $(\mathbb{Z} / 2)^{k}$, Topology 22 (1983), 83-103. MR 84a:55007

[C2] G. Carlsson, Equivariant stable homotopy and Sullivan's conjecture, Invent. Math. 103 (1991), 497-525. MR 86f:57036

[D] D. M. Davis, The BP-coaction for projective spaces, Can. J. Math. 30 (1978), 45-53. MR 57:17640

[H] I. Hansen, Primitive and framed elements in $M U_{*} \mathbb{Z} / p$, Math. Z. 157 (1977), 43-52. MR $\mathbf{5 7 : 4 1 9 9}$

[Hz] M. Hazewinkel, Formal Groups and Applications, Academic Press, New York, 1978. MR 82a: 14020

[JLY] D. C. Johnson, P. S. Landweber and Z. Yoshimura, Injective BP*BP-comodules and localizations of Brown-Peterson homology, Illinois J. Math. 25 (1981), 599-610. MR 82k:55007

[JW] D. C. Johnson and W. S. Wilson, The Brown-Peterson homology of elementary p-groups, Amer. J. Math. 107 (1985), 427-453. MR 86f:55008

[JWY] D. C. Johnson, W. S. Wilson, and D.-Y. Yan, The Brown-Peterson homology of elementary p-groups, II. Topology Appl. 59 (1994), 117-136. MR 95j:55008

[JY] D. C. Johnson and Z. Yosimura, Torsion in Brown-Peterson homology and Hurewicz homomorphisms, Osaka J. Math. 17 (1980), 117-136. MR 81b:55010

[KP] D. S. Kahn and S. B. Priddy, Applications of the transfer to stable homotopy theory, Bull. Amer. Math. Soc. 78 (1972), 981-987. MR 46:8220

[K] M. Kameko, Products of projective spaces as Steenrod modules, Ph. D. Thesis, Johns Hopkins University, May 1990.

[L1] P. S. Landweber, Künneth formulas for bordism theories, Trans. Amer. Math. Soc. 121 (1966), 242-256. MR 33:728

[L2] P. S. Landweber, Coherence, flatness and cobordism of classifying spaces, Proc. Aarhus Summer Institute of algebraic topology, 1970, pp. 256-269.

[Ln] J. Lannes, Sur les espaces fonctionnels dont la source est le classifiant d'un p-groupe abélien élémentaire, Publ. I.H.E.S. 75 (1992), 135-244. MR 93j:55019

[Ll] A. L. Liulevicius, The factorization of cyclic reduced power by secondary cohomology operations, Mem. Amer. Math. Soc., vol. 42, 1962. MR 31:6226

[Ma] M. Mahowald, A new infinite family in ${ }_{2} \pi_{*}^{s}$, Topology 16 (1977), 249-256. MR 56:3838

[Ma2] M. E. Mahowald, Some remarks on the Kervaire invariant problem from the homotopy point of view, Proc. Symp. Pure Math. XXII, Amer. Math. Soc. (1971), 165-169. MR 48:1245

[MM] M. E. Mahowald and R. J. Milgram, Operations that detect $\mathrm{Sq}^{4}$ in connective $K$-theory and their applications, Quart. J. Math. Oxford 27 (1976), 415-432. MR 55:6429

[My] J. P. May, A general algebraic approach to Steenrod operations, Lecture Notes in Mathematics, vol. 168, Springer-Verlag, 1970, pp. 153-231. MR 43:6915

[Ml] H. R. Miller, The Sullivan conjecture on maps from classifying spaces, Annals of Math. 120 (1984), 39-87. MR 85i:55012

[MRW] H. R. Miller, D. C. Ravenel and W. S. Wilson, Periodic phenomena in the Adams-Novikov spectral sequence, Ann. of Math. 106 (1977), 469-516. MR 56:16626

[Mi] J. W. Milnor, The Steenrod algebra and its dual, Ann. of Math. 67 (1958), 150-171. MR 20:6092

[M0] N. Minami, On the double transfer, Algebraic Topology; Oaxtepec 1991 (M. C. Tangora, ed.), Contemp. Math., vol. 146, American Mathematical Society, 1993, pp. 339-347. MR 94e:55024

[M1] N. Minami, The Kervaire invariant one element and the double transfer, Topology 34 (1995), 481-488. MR 95k:55027

[M2] N. Minami, On the odd primary Adams 2-line elements, preprint.

[M3] N. Minami, The Adams spectral sequence and the triple transfer, Amer. J. Math. 117 (1995), 965-985. MR 96d:55010

[Mt] S. A. Mitchell, A proof of the Conner-Floyd conjecture, Amer. J. Math. 106 (1984), 889-891. MR 86k:57030

[N] S. P. Novikov, The methods of algebraic topology from the viewpoint of cobordism theories, Math. USSR-Izv. 31 (1967), 827-913 (Russian), English transl. MR 36:4561 
[P] F. P. Peterson, A-generators for certain polynomial algebras, Math. Proc. Cambridge Phil. Soc. 105 (1989), 311-312. MR 90a:55031

[R1] D. C. Ravenel, Localization with respect to certain periodic homology theories, Amer. J. Math. 106 (1984), 351-414. MR 85k:55009

[R2] D. C. Ravenel, Complex Cobordism and Stable Homotopy Groups of Spheres, Academic Press, Orlando, Florida, 1986, pp. 168-179. MR 87j:55003

[R3] D. C. Ravenel, The geometric realization of the chromatic resolution, Algebraic Topology and Algebraic K-Theory, Annals of Mathematics Studies, vol. 113, Princeton University Press, Princeton, New Jersey, 1987. MR 89d:55050

[RW] D. C. Ravenel and W. S. Wilson, The Morava K-theories of Eilenberg-Mac Lane spaces and the Conner-Floyd conjecture, Amer. J. Math. 102 (1980), 691-748. MR 81i:55005

[Ro] F. W. Roush, Transfer in generalized cohomology theories, Ph. D. thesis, Princeton Univ., 1971.

[S1] W. M. Singer, The transfer in homological algebra, Math. Z. 202 (1989), 493-523. MR 90i: 55035

[S2] W. M. Singer, On the action of Steenrod squares on polynomial algebras, Proc. Amer. Math. Soc. 111 (1991), 577-583. MR 91f:55007

[SX] S. Chen and X. Shen, On the action of Steenrod powers on polynomial algebras, Lecture Notes in Mathematics, vol. 1509, Springer-Verlag, 1992, pp. 326-330. MR 93i:55022

[W] W. S. Wilson, Brown-Peterson Homology, an Introduction and Sampler, Regional Conference Series in Math., No. 48, American Mathematical Society, Providence, Rhode Island, 1980. MR 83j:55005

[Wo] R. M. W. Wood, Steenrod squares of polynomials and the Peterson conjecture, Math. Proc. Cambridge Phil. Soc. 105 (1989), 307-309. MR 90a:55030

Department of Mathematics, The University of Alabama, Box 870350, Tuscaloosa, Alabama 35487-0350

E-mail address: norihiko@gp.as.ua.edu, norihiko@euler.math.ua.edu 